\title{
SHIFT OPERATORS AND TORIC MIRROR THEOREM
}

\author{
HIROSHI IRITANI
}

\begin{abstract}
We give a new proof of Givental's mirror theorem for toric manifolds using shift operators of equivariant parameters. The proof is almost tautological: it gives an A-model construction of the $I$-function and the mirror map. It also works for non-compact or non-semipositive toric manifolds.
\end{abstract}

\section{INTRODUCTION}

In 1995, Seidel Sei97 introduced an invertible element of quantum cohomology associated to a Hamiltonian circle action. This has had many applications in symplectic topology. Seidel himself used it to construct non-trivial elements of $\pi_{1}$ of the group of Hamiltonian diffeomorphisms. McDuff-Tolman [MT06] calculated Seidel's elements in a more general setting and obtained Batyrev's ring presentation of quantum cohomology of toric manifolds. Their method, however, does not yield explicit structure constants of quantum cohomology, i.e. genus-zero Gromov-Witten invariants.

Recently, Braverman, Maulik, Okounkov and Pandharipande [OP10, BMO11, MO12 introduced a shift operator of equivariant parameters for equivariant quantum cohomology. Their shift operators reduce to Seidel's invertible elements under the nonequivariant limit. In this paper, we show that equivariant genus-zero Gromov-Witten invariants of toric manifolds are reconstructed only from formal properties of shift operators. This means that the equivariant quantum topology of toric manifolds is determined by its classical counterpart.

More specifically, we give a new proof of Givental's mirror theorem for toric manifolds, which is stated as follows:

Theorem 1.1 ([Giv981, [LY99, [ri08, Bro09], see $\$ 4.2$ for more details). Let $X_{\Sigma}$ be a semi-projective toric manifold having a torus fixed point. Let $I(y, z)$ be the cohomologyvalued hypergeometric series defined by

$$
I(y, z)=z e^{\sum_{i=1}^{m} u_{i} \log y_{i} / z} \sum_{d \in \operatorname{Eff}\left(X_{\Sigma}\right)}\left(\prod_{i=1}^{m} \frac{\prod_{c=-\infty}^{0}\left(u_{i}+c z\right)}{\prod_{c=-\infty}^{u_{i} \cdot d}\left(u_{i}+c z\right)}\right) Q^{d} y_{1}^{u_{1} \cdot d} \cdots y_{m}^{u_{m} \cdot d}
$$

where $u_{i}, i=1, \ldots, m$ is the class of a prime toric divisor. Then $I(y,-z)$ lies in Givental's Lagrangian cone $\mathcal{L}_{X_{\Sigma}}$ associated to $X_{\Sigma}$.

We prove this theorem in the following way. Recall that equivariant genus-zero Gromov-Witten invariants of a $T$-variety $X$ can be encoded by an infinite-dimensional Lagrangian submanifold $\mathcal{L}_{X}$ of the symplectic vector space $\mathcal{H}_{X}$ Giv04:

$$
\mathcal{H}_{X}=H_{T}^{*}(X) \otimes_{H_{T}^{*}(\mathrm{pt})} \operatorname{Frac}\left(H_{T}^{*}(\mathrm{pt})[z]\right) .
$$


The space $\mathcal{H}_{X}$ is called the Givental space and $\mathcal{L}_{X}$ is called the Givental cone. By the general theory, each $\mathbb{C}^{\times}$-subgroup $k: \mathbb{C}^{\times} \rightarrow T$ defines a shift operator $\mathcal{S}_{k}$ acting on the Givental space $\mathcal{H}_{X}$ and induces a vector field on $\mathcal{L}_{X}$ :

$$
\mathcal{L}_{X} \ni \mathbf{f} \longmapsto z^{-1} \mathcal{S}_{k} \mathbf{f} \in T_{\mathbf{f}} \mathcal{L}_{X}
$$

The operator $\mathcal{S}_{k}$ is determined by $T$-fixed loci in $X$ and their normal bundles (see Definition 3.13). For toric manifolds, we have a shift operator $\mathcal{S}_{i}$ for each torus-invariant prime divisor. Then we identify the $I$-function $I(y, z)$ with an integral curve of the commuting vector fields $\mathbf{f} \mapsto z^{-1} \mathcal{S}_{i} \mathbf{f}$.

Theorem 1.2. Givental's I-function $I(y, z)$ is a unique integral curve which satisfies the differential equation:

$$
\frac{\partial I(y, z)}{\partial y_{i}}=z^{-1} \mathcal{S}_{i} I(y, z) \quad i=1, \ldots, m
$$

and is of the form $I(y, z)=z e^{\sum_{i=1}^{m} u_{i} \log y_{i} / z}\left(1+\sum_{d \in \operatorname{Eff}\left(X_{\Sigma}\right) \backslash\{0\}} I_{d} Q^{d} y^{d}\right)$, where we set $y^{d}=\prod_{i=1}^{m} y_{i}^{u_{i} \cdot d}$.

The $I$-function defines a mirror map $y \mapsto \tau(y) \in H_{T}^{*}(X)$ via Birkhoff factorization CG07, Iri08. As a corollary to our proof, we obtain the following relationship between the equivariant Seidel elements $S_{i}(\tau)$ and the mirror map. This generalizes a previous result GI12 in the semipositive case obtained in joint work with Gonzalez.

Theorem 1.3. The mirror map $\tau(y)$ associated to the I-function is a unique integral curve which satisfies the differential equation

$$
\frac{\partial \tau(y)}{\partial y_{i}}=S_{i}(\tau(y)) \quad i=1, \ldots, m
$$

and is of the form $\tau(y)=\sum_{i=1}^{m} u_{i} \log y_{i}+\sum_{d \in \operatorname{Eff}\left(X_{\Sigma}\right) \backslash\{0\}} \tau_{d} Q^{d} y^{d}$.

The mirror map and the $I$-function are related by the formula

$$
I(y, z)=z M(\tau(y), z) \Upsilon(y, z)
$$

where $M(\tau, z)$ is a fundamental solution for the quantum differential equation (Proposition 2.2) and $\Upsilon(y, z)$ is an $H_{T}^{*}(X)[z]$-valued function. We can also characterize $\Upsilon(y, z)$ by the differential equation

$$
\frac{\partial \Upsilon(y, z)}{\partial y_{i}}=\left[z^{-1} \mathbb{S}_{i}(\tau(y))\right]_{+} \Upsilon(y, z)
$$

where $\mathbb{S}_{i}(\tau)$ is the shift operator acting on quantum cohomology. The most technical point in our proof is to show the existence of solutions $\tau(y)$ and $\Upsilon(y, z)$ with prescribed asymptotics (see Proposition 4.7).

Since we do not assume that $c_{1}\left(X_{\Sigma}\right)$ is nef, the mirror map $\tau(y)$ does not necessarily lie in $H_{T}^{\leq 2}(X)$. For this reason, we need to generalize shift operators to big quantum cohomology. We also observe that shift operators are closely related to the $\widehat{\Gamma}$-integral structure [Iri09, KKP08, CIJ14]. We show that a flat section of the quantum connection associated to an equivariant vector bundle in the formalism of $\widehat{\Gamma}$-integral structure is invariant under shift operators (Proposition 3.18). 
This paper is structured as follows. In 32 , we review equivariant quantum cohomology and in $\S 3$, we study shift operators for big quantum cohomology. In $\S$, we prove a mirror theorem for toric manifolds.

1.1. Notation. Unless otherwise stated, we consider cohomology groups with complex coefficients. We use the following notation throughout the paper.

- $T \cong\left(\mathbb{C}^{\times}\right)^{m}:$ an algebraic torus;

- $X$ : a smooth $T$-variety; $X_{\Sigma}$ : a smooth toric variety associated to a fan $\Sigma$;

- $\widehat{T}=T \times \mathbb{C}^{\times}$;

- $\lambda \in \operatorname{Lie}(T), z \in \operatorname{Lie}\left(\mathbb{C}^{\times}\right)$: equivariant parameters for $\widehat{T}$;

- $H_{\widehat{T}}(X)_{\text {loc }}:=H_{\widehat{T}}^{*}(X) \otimes_{H_{\widehat{T}}^{*}(\mathrm{pt})} \operatorname{Frac}\left(H_{\widehat{T}}^{*}(\mathrm{pt})\right)=H_{T}^{*}(X) \otimes_{H_{T}^{*}(\mathrm{pt})} \operatorname{Frac}\left(H_{T}^{*}(\mathrm{pt})[z]\right)$ : the Givental space.

Acknowledgments. The author thanks Tom Coates, Alessio Corti, Eduardo Gonzalez, Hiraku Nakajima and Hsian-Hua Tseng for very helpful discussions on shift operators, Seidel representations and toric mirror symmetry. This work is supported by JSPS Kakenhi Grant Number 25400069.

\section{Equivariant Quantum Cohomology}

2.1. Hypotheses on a $T$-Space. Let $T \cong\left(\mathbb{C}^{\times}\right)^{m}$ be an algebraic torus. Let $X$ be a smooth variety over $\mathbb{C}$ equipped with an algebraic $T$-action. We assume the following conditions:

(1) $X$ is semi-projective, i.e. the natural map $X \rightarrow X_{0}:=\operatorname{Spec} H^{0}(X, \mathcal{O})$ is projective;

(2) all $T$-weights appearing in the $T$-representation $H^{0}(X, \mathcal{O})$ are contained in a strictly convex cone in $\operatorname{Hom}\left(T, \mathbb{C}^{\times}\right) \otimes \mathbb{R}$ and $H^{0}(X, \mathcal{O})^{T}=\mathbb{C}$.

A $T$-space $X$ with these assumptions has nice cohomological properties, see, e.g. HRV13. These conditions ensure that the $T$-fixed set $X^{T}$ is projective. We also note the following:

Proposition 2.1. A smooth $T$-variety $X$ satisfying the conditions (11), (2) is equivariantly formal, i.e. $H_{T}^{*}(X)$ is a free module over $H_{T}^{*}(\mathrm{pt})$ and there is a non-canonical isomorphism $H_{T}^{*}(X) \cong H^{*}(X) \otimes H_{T}^{*}(\mathrm{pt})$ as an $H_{T}^{*}(\mathrm{pt})$-module.

Proof. We use the argument of Kirwan [Kir84, Proposition 5.8] (see also [Nak99, §5.1]). Choose a one-parameter subgroup $k: \mathbb{C}^{\times} \rightarrow T$ such that $k$ is negative on every nonzero weight of $H^{0}(X, \mathcal{O})$. This defines a $\mathbb{C}^{\times}$-action on $X$. Let $L \rightarrow X$ be a very ample line bundle. The $\mathbb{C}^{\times}$-action on $X$ lifts to a $\mathbb{C}^{\times}$-linearization on $L$, possibly after replacing $L$ with its power $L^{\otimes i}$ Dol03, Corollary 7.2]. Then $L$ defines a $\mathbb{C}^{\times}$-equivariant closed embedding $X \hookrightarrow X_{0} \times \mathbb{P}^{n}$, where $\mathbb{P}^{n}$ is equipped with a linear $\mathbb{C}^{\times}$-action. By assumption, we can embed the affine variety $X_{0}=\operatorname{Spec}\left(H^{0}(X, \mathcal{O})\right)$ equivariantly into a $\mathbb{C}^{\times}$-representation $V$ which has only positive $\|^{\infty}$ weights. Thus we have a $\mathbb{C}^{\times}$-equivariant closed embedding $X \hookrightarrow V \times \mathbb{P}^{n}$. The associated $S^{1}$-action on $V \times \mathbb{P}^{n}$ admits, with respect to the standard Kähler metric, a moment map $\mu$ which is proper and bounded

\footnotetext{
${ }^{1}$ We use the (usual) convention that $t \in \mathbb{C}^{\times}$acts on functions by $f(x) \mapsto f\left(t^{-1} x\right)$.
} 
from below. These properties allow us to use Morse theory for the moment map $\left.\mu\right|_{X}$. The argument in Kir84, Nak99 shows that $\left.\mu\right|_{X}$ is a perfect Bott-Morse function and $X$ is equivariantly formal.

2.2. Gromov-Witten Invariants. For a second homology class $d \in H_{2}(X, \mathbb{Z})$ and a non-negative integer $n \geq 0$, we denote by $X_{0, n, d}$ the moduli stack of genus-zero stable maps to $X$ of degree $d$ with $n$ marked points. The $T$-action on $X$ induces a $T$-action on $X_{0, n, d}$. It has a virtual fundamental class $\left[X_{0, n, d}\right]_{\text {vir }} \in H_{*}\left(X_{0, n, d}, \mathbb{Q}\right)$ of dimension $D=$ $\operatorname{dim} X+n-3+c_{1}(X) \cdot d$. For equivariant cohomology classes $\alpha_{1}, \ldots, \alpha_{n} \in H_{T}^{*}(X, \mathbb{Q})$ and non-negative integers $k_{1}, \ldots, k_{n}$, the genus-zero $T$-equivariant Gromov-Witten invariant is defined by

$$
\left\langle\alpha_{1} \psi^{k_{1}}, \ldots, \alpha_{n} \psi^{k_{n}}\right\rangle_{0, n, d}^{X, T}=\int_{\left[X_{0, n, d}\right]_{\mathrm{vir}}} \prod_{i=1}^{n} \mathrm{ev}_{i}^{*}\left(\alpha_{i}\right) \psi_{i}^{k_{i}} .
$$

Here $\mathrm{ev}_{i}: X_{0, n, d} \rightarrow X$ is the evaluation map at the $i$ th marked point and $\psi_{i}$ denotes the equivariant first Chern class of the $i$ th universal cotangent line bundle $L_{i}$ over $X_{0, n, d}$. When the moduli space $X_{0, n, d}$ is not compact, the right-hand side is defined via the Atiyah-Bott localization formula AB84, GP99 and belongs to the fraction field $\operatorname{Frac}\left(H_{T}^{*}(\mathrm{pt})\right)$ of $H_{T}^{*}(\mathrm{pt})$.

2.3. Quantum Cohomology. Let $\operatorname{Eff}(X) \subset H_{2}(X, \mathbb{Z})$ denote the semigroup of homology classes of effective curves. We write $Q$ for the Novikov variable and define $M \llbracket Q \rrbracket$ to be the space of formal power series:

$$
M \llbracket Q \rrbracket=\left\{\sum_{d \in \operatorname{Eff}(X)} a_{d} Q^{d}: a_{d} \in M\right\}
$$

with coefficients in a module $M$. When $M$ is a ring, $M \llbracket Q \rrbracket$ is also a ring. Let $(\cdot, \cdot)$ denote the $T$-equivariant Poincaré pairing on $H_{T}^{*}(X)$ :

$$
(\alpha, \beta)=\int_{X} \alpha \cup \beta
$$

If $X$ is not compact, we define the right-hand side via the localization formula. Therefore $(\cdot, \cdot)$ takes values in $\operatorname{Frac}\left(H_{T}^{*}(\mathrm{pt})\right)$ in general. Let $\left\{\phi_{i}\right\}_{i=0}^{N}$ be a basis of $H_{T}^{*}(X)$ over $H_{T}^{*}(\mathrm{pt})$. We write $\left\{\tau^{i}\right\}_{i=0}^{N}$ for the dual co-ordinates on $H_{T}^{*}(X)$ and $\tau=\sum_{i=0}^{N} \tau^{i} \phi_{i}$ for a general point on $H_{T}^{*}(X)$. The (big) quantum product $\star$ is defined by the formula

$$
\left(\phi_{i} \star \phi_{j}, \phi_{k}\right)=\sum_{d \in \operatorname{Eff}(X)} \sum_{n=0}^{\infty} \frac{Q^{d}}{n !}\left\langle\phi_{i}, \phi_{j}, \phi_{k}, \tau, \ldots, \tau\right\rangle_{0, n+3, d}^{X, T} .
$$

We note that the quantum product $\phi_{i} \star \phi_{j}$ is defined without localization:

$$
\phi_{i} \star \phi_{j} \in H_{T}^{*}(X) \llbracket Q \rrbracket \llbracket \tau^{0}, \ldots, \tau^{N} \rrbracket .
$$

In fact, $\phi_{i} \star \phi_{j}$ can be written as the push-forward

$$
\sum_{d \in \operatorname{Eff}(X)} \sum_{n=0}^{\infty} \frac{Q^{d}}{n !} \operatorname{PD~ev}_{3 *}\left(\operatorname{ev}_{1}^{*}\left(\phi_{i}\right) \operatorname{ev}_{2}^{*}\left(\phi_{j}\right) \prod_{l=4}^{n+3} \operatorname{ev}_{l}^{*}(\tau) \cap\left[X_{0, n+3, d}\right]_{\text {vir }}\right)
$$


along the proper evaluation map $\mathrm{ev}_{3}$, and hence the localization is not necessary. The properness of $\mathrm{ev}_{3}$ follows from the assumption that $X$ is semi-projective.

2.4. Quantum Connection and Fundamental Solution. The quantum connection is the operator

$$
\nabla_{i}: H_{T}^{*}(X)[z] \llbracket Q \rrbracket \llbracket \tau^{0}, \ldots, \tau^{N} \rrbracket \rightarrow z^{-1} H_{T}^{*}(X)[z] \llbracket Q \rrbracket \llbracket \llbracket \tau^{0}, \ldots, \tau^{N} \rrbracket
$$

defined by

$$
\nabla_{i}=\frac{\partial}{\partial \tau^{i}}+\frac{1}{z}\left(\phi_{i} \star\right)
$$

The quantum connection has a parameter $z$ : we identify it with the equivariant parameter for an additional $\mathbb{C}^{\times}$-action. Set $\widehat{T}=T \times \mathbb{C}^{\times}$and consider the $\widehat{T}$-action on $X$ induced by the projection $\widehat{T} \rightarrow T$. Then we have $H_{\widehat{T}}^{*}(X) \cong H_{T}^{*}(X)[z]$. The quantum connection is known to be flat, and admits a fundamental solution:

$$
M(\tau): H_{\widehat{T}}^{*}(X) \llbracket Q \rrbracket \llbracket \tau^{0}, \ldots, \tau^{N} \rrbracket \rightarrow H_{\widehat{T}}^{*}(X)_{\mathrm{loc}} \llbracket Q \rrbracket \llbracket \tau^{0}, \ldots, \tau^{N} \rrbracket
$$

satisfying the quantum differential equation:

$$
z \frac{\partial}{\partial \tau^{i}} M(\tau)=M(\tau)\left(\phi_{i} \star\right)
$$

or equivalently $\left(\partial / \partial \tau^{i}\right) \circ M(\tau)=M(\tau) \circ \nabla_{i}$, where $H_{\widehat{T}}^{*}(X)_{\text {loc }}:=H_{\widehat{T}}^{*}(X) \otimes_{H_{\widehat{T}}^{*}(\mathrm{pt})}$ $\operatorname{Frac}\left(H_{\widehat{T}}^{*}(\mathrm{pt})\right)$ is the localized equivariant cohomology. The following proposition is well-known, see Giv98a, §1], [Pan98, Proposition 2].

Proposition 2.2. A fundamental solution is given by

$$
\left(M(\tau) \phi_{i}, \phi_{j}\right)=\left(\phi_{i}, \phi_{j}\right)+\sum_{\substack{d \in \operatorname{Eff}(X), n \geq 0 \\(d, n) \neq(0,0)}} \frac{Q^{d}}{n !}\left\langle\phi_{i}, \tau, \ldots, \tau, \frac{\phi_{j}}{z-\psi}\right\rangle_{0, n+2, d}^{X, T}
$$

Remark 2.3. Expanding $1 /(z-\psi)=\sum_{n=0}^{\infty} \psi^{n} / z^{n+1}$, we find that $M(\tau) \phi_{i}$ takes values in $H_{T}^{*}(X) \llbracket z^{-1} \rrbracket$. By the localization calculation, it also follows that $M(\tau) \phi_{i}$ takes values in $H_{\widehat{T}}^{*}(X)_{\text {loc }}$. The localized $\widehat{T}$-equivariant cohomology $H_{\widehat{T}}^{*}(X)_{\text {loc }}$ is also called the Givental space Giv04.

\section{Shift Operator}

The shift operator for equivariant quantum cohomology has been introduced by Okounkov-Pandharipande [OP10], Braverman-Maulik-Okounkov [BMO11] and MaulikOkounkov MO12]. We discuss its (straightforward) extension to the big quantum cohomology. 
3.1. Twisted Homomorphism. We write $\widehat{T}=T \times \mathbb{C}^{\times}$. For a group homomorphism $k: \mathbb{C}^{\times} \rightarrow T$, we consider the $\widehat{T}$-action $\rho_{k}$ on $X$ defined by

$$
\rho_{k}(t, u) x=t u^{k} \cdot x
$$

where $(t, u) \in \widehat{T}, x \in X$ and $u^{k} \in T$ denotes the image of $u \in \mathbb{C}^{\times}$under $k$. Let $\lambda \in \operatorname{Lie}(T)$ denote the equivariant parameter for $T$ and let $z \in \operatorname{Lie}\left(\mathbb{C}^{\times}\right)$denote the equivariant parameter for $\mathbb{C}^{\times}$. The identity map id: $\left(X, \rho_{0}\right) \rightarrow\left(X, \rho_{k}\right)$ is equivariant with respect to the group automorphism

$$
\phi_{k}: \widehat{T} \rightarrow \widehat{T}, \quad \phi_{k}(t, u)=\left(t u^{-k}, u\right) .
$$

Therefore the identity map induces an isomorphism

$$
\Phi_{k}: H_{\widehat{T}, \rho_{0}}^{*}(X) \cong H_{\widehat{T}, \rho_{k}}^{*}(X)
$$

such that

$$
\Phi_{k}(f(\lambda, z) \alpha)=f(\lambda+k z, z) \Phi_{k}(\alpha)
$$

where $\alpha \in H_{\widehat{T}, \rho_{0}}^{*}(X)$ and $f(\lambda, z) \in H_{\widehat{T}}^{*}(\mathrm{pt})$ is a polynomial function on $\operatorname{Lie}(\widehat{T})$. Referring to the property (3.1), we say that $\Phi_{k}$ is a $k$-twisted homomorphism.

Notation 3.1. We write $H_{\widehat{T}, \rho}^{*}(X)$ for the $\widehat{T}$-equivariant cohomology of $X$ with respect to the $\widehat{T}$-action $\rho$ on $X$. When $\rho$ is omitted, $H_{\widehat{T}}^{*}(X)$ means $H_{\widehat{T}, \rho_{0}}^{*}(X)$.

\subsection{Bundle Associated to a $\mathbb{C}^{\times}$-Subgroup.}

Definition 3.2 (associated bundle). Let $k: \mathbb{C}^{\times} \rightarrow T$ be a group homomorphism. Consider the $\mathbb{C}^{\times}$-action on $X \times\left(\mathbb{C}^{2} \backslash\{0\}\right)$ given by $s \cdot\left(x,\left(v_{1}, v_{2}\right)\right)=\left(s^{k} \cdot x,\left(s^{-1} v_{1}, s^{-1} v_{2}\right)\right)$. Let $E_{k}$ denote the quotient space:

$$
E_{k}:=X \times\left(\mathbb{C}^{2} \backslash\{0\}\right) / \mathbb{C}^{\times} .
$$

We have a natural projection $\pi: E_{k} \rightarrow \mathbb{P}^{1}$ given by $\pi\left(\left[x,\left(v_{1}, v_{2}\right)\right]\right)=\left[v_{1}, v_{2}\right]$ and $E_{k}$ is a fiber bundle over $\mathbb{P}^{1}$ with fiber $X$. We consider the $\widehat{T}$-action on $E_{k}$ given by $(t, u) \cdot\left[x,\left(v_{1}, v_{2}\right)\right]=\left[t \cdot x,\left(v_{1}, u v_{2}\right)\right]$. Let $X_{0}$ denote the fiber of $E_{k} \rightarrow \mathbb{P}^{1}$ at $[1,0]$ and let $X_{\infty}$ denote the fiber at $[0,1]$. Note that we have

$$
X_{0} \cong\left(X, \rho_{0}\right) \quad \text { and } \quad X_{\infty} \cong\left(X, \rho_{k}\right)
$$

as $\widehat{T}$-spaces.

Definition 3.3. A group homomorphism $k: \mathbb{C}^{\times} \rightarrow T$ is said to be semi-negative if $k$ is non-positive on each $T$-weight of $H^{0}(X, \mathcal{O})$. We say that $k$ is negative if $k$ is negative on each non-zero $T$-weight of $H^{0}(X, \mathcal{O})$.

Remark 3.4. When $X$ is complete, every $\mathbb{C}^{\times}$-subgroup is negative.

Suppose that $k: \mathbb{C}^{\times} \rightarrow T$ is semi-negative and consider the $\mathbb{C}^{\times}$-action on $X$ induced by $k$. Let $L$ be a very ample line bundle on $X$. As discussed in the proof of Proposition 2.1, we may assume that $L$ admits a $\mathbb{C}^{\times}$-linearization. By tensoring $L$ with a $\mathbb{C}^{\times}$character, we may assume that all the $\mathbb{C}^{\times}$-weights on $H^{0}\left(X, L^{\otimes n}\right)$ are negative for $n>0$. Let $p: X \times \mathbb{C}^{2} \rightarrow X$ be the natural projection. Then $p^{*} L$ is a $\mathbb{C}^{\times}$-equivariant line bundle 
on $X \times \mathbb{C}^{2}$, where $\mathbb{C}^{\times}$acts on the base by $s \cdot\left(x,\left(v_{1}, v_{2}\right)\right)=\left(s^{k} \cdot x,\left(s^{-1} v_{1}, s^{-1} v_{2}\right)\right)$. We can see that

$$
H^{0}\left(X \times \mathbb{C}^{2},\left(p^{*} L\right)^{\otimes n}\right)=\bigoplus_{i=0}^{\infty} H^{0}\left(X, L^{\otimes n}\right)^{(-i)} \otimes \mathbb{C}\left[v_{1}, v_{2}\right]^{(i)}
$$

where the superscript $(l)$ means the component of $\mathbb{C}_{s}^{\times}$-weight $l$. The unstable locus for the $\mathbb{C}^{\times}$-action on $\left(X \times \mathbb{C}^{2}, p^{*} L\right)$, in the sense of Geometric Invariant Theory (GIT), is $X \times\{0\}$ and therefore we find that $E_{k}$ is the GIT quotient of $X \times \mathbb{C}^{2}$, i.e. $E_{k}=$ $\operatorname{Proj}\left(\bigoplus_{n=0}^{\infty} H^{0}\left(X \times \mathbb{C}^{2},\left(p^{*} L\right)^{\otimes n}\right)\right)$. This proves:

Lemma 3.5. If $k$ is semi-negative, $E_{k}$ is semi-projective.

Let $k: \mathbb{C}^{\times} \rightarrow T$ be a semi-negative subgroup and consider the $\mathbb{C}^{\times}$-action on $X$ induced by $k$. A $\mathbb{C}^{\times}$-fixed point $x \in X$ defines a section of $E_{k} \rightarrow \mathbb{P}^{1}$ :

$$
\sigma_{x}=\left(\{x\} \times \mathbb{P}^{1}\right) \subset E_{k} .
$$

We now define a minimal section among all such sections associated to fixed points. Using the argument in the proof of Proposition 2.1, we obtain a $\mathbb{C}^{\times}$-equivariant closed embedding $X \hookrightarrow \mathbb{P}^{n} \times \mathbb{C}^{l}$ where $\mathbb{C}^{l}$ is a $\mathbb{C}^{\times}$-representation with only non-negative weights. In particular, for every point $x \in X$, the $\operatorname{limit}_{\lim _{s \rightarrow 0}} s^{k} \cdot x$ exists. This implies the existence of the Bialynicki-Birula decomposition [BB73, Theorem 4.1] for $X$ : if $X^{\mathbb{C}^{\times}}=\bigsqcup_{i} F_{i}$ is the decomposition of the $\mathbb{C}^{\times}$-fixed locus $X^{\mathbb{C}^{\times}}$into connected components, we have the induced decomposition of $X$

$$
X=\bigsqcup_{i} U_{i}, \quad U_{i}=\left\{x \in X: \lim _{s \rightarrow 0} s^{k} \cdot x \in F_{i}\right\}
$$

into locally closed smooth subvarieties $U_{i}$. In particular there exists a unique $\mathbb{C}^{\times}$-fixed component $F_{\min } \subset X$ such that all the $\mathbb{C}^{\times}$-weights on the normal bundle to $F_{\text {min }}$ are positive. The moment map $\mu$ for the associated $S^{1}$-action attains a global minimum on $F_{\text {min }}$. We call the class of a section $\sigma_{\min }$ of $E_{k}$ associated to a point in $F_{\min }$ the minimal section class. We write

$$
\begin{aligned}
H_{2}^{\mathrm{sec}}\left(E_{k}, \mathbb{Z}\right) & =\left\{d \in H_{2}\left(E_{k}, \mathbb{Z}\right): \pi_{*}(d)=\left[\mathbb{P}^{1}\right]\right\}, \\
\operatorname{Eff}\left(E_{k}\right)^{\mathrm{sec}} & =\operatorname{Eff}\left(E_{k}\right) \cap H_{2}^{\mathrm{sec}}\left(E_{k}, \mathbb{Z}\right) .
\end{aligned}
$$

Lemma 3.6. If $k$ is semi-negative, we have $\operatorname{Eff}\left(E_{k}\right)^{\mathrm{sec}}=\sigma_{\min }+\operatorname{Eff}(X)$.

Proof. The compact case was discussed in [GI12, Lemma 2.2]. Take a negative oneparameter subgroup $l: \mathbb{C}^{\times} \rightarrow T$ and consider the $\mathbb{C}^{\times}$-action on $E_{k}$ induced by $\mathbb{C}^{\times} \stackrel{l}{\rightarrow}$ $T \times\{1\} \subset \widehat{T}$. Observe that all non-zero $\mathbb{C}^{\times}$-weights on $H^{0}\left(E_{k}, \mathcal{O}\right)$ are negative. This means that $E_{k, 0}:=\operatorname{Spec} H^{0}\left(E_{k}, \mathcal{O}\right)$ has a unique $\mathbb{C}^{\times}$-fixed point 0 and $\lim _{s \rightarrow 0} s \cdot x=0$ for all $x \in E_{k, 0}$. Therefore every curve can be deformed, via the $\mathbb{C}^{\times}$-action, to a stable curve in the fiber $K$ of $E_{k} \rightarrow E_{k, 0}$ at $0 \in E_{k, 0}$ in the same homology class. Since $\widehat{T}$-action on $E_{k}$ preserves $K$ and $K$ is compact, we may further deform a curve in $K$ to a $\widehat{T}$-invariant stable curve. A $\widehat{T}$-invariant stable curve in $E_{k}$ is a union of a section class $\sigma_{x}$ associated to a $T$-fixed point $x \in X$ and effective curves in $X_{0} \sqcup X_{\infty}$. Suppose that two different fixed points $x, y \in X^{T}$ are connected by a $k\left(\mathbb{C}^{\times}\right)$-orbit, i.e. $\exists p \in X$, 
$x=\lim _{s \rightarrow \infty} s^{k} \cdot p$ and $y=\lim _{s \rightarrow 0} s^{k} \cdot p$. The closure $C=\overline{k\left(\mathbb{C}^{\times}\right) \cdot p}$ is isomorphic to $\mathbb{P}^{1}$ and $\sigma_{x}, \sigma_{y}$ are contained in a Hirzebruch surface

$$
C \times\left(\mathbb{C}^{2} \backslash\{0\}\right) / \mathbb{C}^{\times} \subset E_{k}
$$

Then one finds $\sigma_{x}=\sigma_{y}+a[C]$ for some $a>0$. Using the Bialynicki-Birula decomposition for the $k\left(\mathbb{C}^{\times}\right)$-action on $X$, we find that every $T$-fixed point is connected to a $T$-fixed point on $F_{\min }$ by a chain of $k\left(\mathbb{C}^{\times}\right)$-orbits. The conclusion follows.

Lemma 3.7. We have an isomorphism

$$
H_{\widehat{T}}^{*}\left(E_{k}\right) \cong\left\{(\alpha, \beta) \in H_{\widehat{T}, \rho_{0}}^{*}(X) \oplus H_{\widehat{T}, \rho_{k}}^{*}(X): \alpha-\Phi_{k}^{-1}(\beta) \equiv 0 \quad \bmod z\right\}
$$

which sends $\tau$ to $\left(\left.\tau\right|_{X_{0}},\left.\tau\right|_{X_{\infty}}\right)$. Recall that $z$ is the equivariant parameter for $\mathbb{C}^{\times}$and we have a canonical isomorphism $H_{\widehat{T}, \rho_{0}}^{*}(X) \cong H_{T}^{*}(X)[z]$.

Proof. Consider the Mayer-Vietoris exact sequence associated to the covering $E_{k}=$ $U_{0} \cup U_{\infty}$ with $U_{0}=\pi^{-1}(\mathbb{C})$ and $U_{\infty}=\pi^{-1}\left(\mathbb{P}^{1} \backslash\{0\}\right)$. We have $H_{\widehat{T}}^{*}\left(U_{0}\right) \cong H_{\widehat{T}, \rho_{0}}^{*}(X)$, $H_{\widehat{T}}^{*}\left(U_{\infty}\right) \cong H_{\widehat{T}, \rho_{k}}^{*}(X)$ and $H_{\widehat{T}}^{*}\left(U_{0} \cap U_{\infty}\right) \cong H_{T}(X)$. The map $H_{\widehat{T}}^{*}\left(U_{0}\right) \oplus H_{\widehat{T}}^{*}\left(U_{\infty}\right) \rightarrow$ $H_{\widehat{T}}^{*}\left(U_{0} \cap U_{\infty}\right)$ is surjective and is given by $\left.(\alpha, \beta) \mapsto\left(\alpha-\Phi_{k}^{-1} \beta\right)\right|_{z=0}$.

Notation 3.8. By Lemma 3.7, for $\tau \in H_{T}^{*}(X)$, there exists $\hat{\tau} \in H_{\widehat{T}}^{*}\left(E_{k}\right)$ such that $\left.\hat{\tau}\right|_{X_{0}}=\tau$ and $\left.\hat{\tau}\right|_{X_{\infty}}=\Phi_{k}(\tau)$. This defines a map ${ }^{\wedge}: H_{T}^{*}(X) \rightarrow H_{\widehat{T}}^{*}\left(E_{k}\right)$. This is not $H_{T}^{*}(\mathrm{pt})$-linear.

\subsection{Shift Operator.}

Definition 3.9 (shift operator). Let $k: \mathbb{C}^{\times} \rightarrow T$ be a semi-negative group homomorphism. For $\tau \in H_{T}^{*}(X)$, we define $\widetilde{\mathbb{S}}_{k}(\tau): H_{\widehat{T}, \rho_{0}}^{*}(X) \llbracket Q \rrbracket \rightarrow H_{\widehat{T}, \rho_{k}}^{*}(X) \llbracket Q \rrbracket$ by

$$
\left(\widetilde{\mathbb{S}}_{k}(\tau) \alpha, \beta\right)=\sum_{\hat{d} \in \operatorname{Eff}\left(E_{k}\right)^{\mathrm{sec}}} \frac{Q^{\hat{d}-\sigma_{\min }}}{n !}\left\langle\iota_{0 *} \alpha, \iota_{\infty *} \beta, \hat{\tau}, \ldots, \hat{\tau}\right\rangle_{0, n+2, \hat{d}}^{E_{k}, \widehat{T}}
$$

where $(\cdot, \cdot)$ in the left-hand side is the $\widehat{T}$-equivariant Poincaré pairing on $H_{\widehat{T}, \rho_{k}}^{*}(X)$, $\alpha \in H_{\widehat{T}, \rho_{0}}^{*}(X), \beta \in H_{\widehat{T}, \rho_{k}}^{*}(X), \sigma_{\min }$ is the minimal section class for $E_{k}$, and $\iota_{0}: X_{0} \rightarrow E_{k}$, $\iota_{\infty}: X_{\infty} \rightarrow E_{k}$ are the natural inclusions. We also define

$$
\mathbb{S}_{k}(\tau)=\Phi_{k}^{-1} \circ \widetilde{\mathbb{S}}_{k}(\tau): H_{\widehat{T}}^{*}(X) \llbracket Q \rrbracket \rightarrow H_{\widehat{T}}^{*}(X) \llbracket Q \rrbracket .
$$

Note that $\widetilde{\mathbb{S}}_{k}$ is untwisted but $\mathbb{S}_{k}$ is $(-k)$-twisted (see (3.1)).

Remark 3.10. When $k$ is semi-negative, $E_{k}$ is semi-projective by Lemma 3.5 and thus the shift operator $\mathbb{S}_{k}$ is defined without localization: we may rewrite $\widetilde{\mathbb{S}}_{k}$ as the pushforward along an evaluation map (see (2.1)). When $k$ is not semi-negative, we can still define $\mathbb{S}_{k}$ over $\operatorname{Frac}\left(H_{T}^{*}(\mathrm{pt})\right)$ after choosing a suitable section class $\sigma_{\min }$.

Remark 3.11. Since the map $\tau \mapsto \hat{\tau}$ is not $H_{T}^{*}(\mathrm{pt})$-linear, $\mathbb{S}(\tau)$ cannot be written as formal power series in the $H_{T}^{*}(\mathrm{pt})$-valued variables $\tau^{0}, \ldots, \tau^{N}$. For $\alpha_{1}, \ldots, \alpha_{l} \in H_{T}^{*}(X)$ and $\mathbb{C}$-valued variables $t^{1}, \ldots, t^{l}$, the shift operator $\mathbb{S}(\tau)$ with $\tau=\sum_{i=1}^{l} t^{i} \alpha_{i}$ is a formal power series in $t^{1}, \ldots, t^{l}$. 
Remark 3.12 (divisor equation). Suppose that $\tau=h+\tau^{\prime}$ with $h \in H_{T}^{2}(X)$. Using the divisor equation, we have:

$$
\left(\widetilde{\mathbb{S}}_{k}(\tau) \alpha, \beta\right)=e^{-h(k)} \sum_{d \in \operatorname{Eff}(X)} \frac{Q^{d} e^{h \cdot d}}{n !}\left\langle\iota_{0 *} \alpha, \iota_{\infty *} \beta, \hat{\tau}^{\prime}, \ldots \hat{\tau}^{\prime}\right\rangle_{0, n+2, \sigma_{\min }+d}^{E_{k}, \widehat{T}}
$$

where $h(k)$ is the pairing between $k$ and the restriction $\left.h\right|_{x} \in H_{T}^{2}(\mathrm{pt}) \cong \operatorname{Lie}(T)^{*}$ of $h$ to a fixed point $x$ in the minimal fixed component $F_{\min }$ (with respect to $k$ ). Note that $\hat{h} \cdot \sigma_{\min }=-h(k)$.

By the localization theorem of equivariant cohomology [AB84, the restriction to the $T$-fixed subspace $X^{T}$ induces an isomorphism

$$
\iota^{*}: H_{\widehat{T}}^{*}(X)_{\mathrm{loc}} \stackrel{\cong}{\longrightarrow} H_{\widehat{T}}^{*}\left(X^{T}\right)_{\mathrm{loc}}=H^{*}\left(X^{T}\right) \otimes \operatorname{Frac}\left(H_{\widehat{T}}^{*}(\mathrm{pt})\right) .
$$

We use this to define the shift operator on the Givental space $H_{\widehat{T}}^{*}(X)_{\text {loc }}$.

Definition 3.13 (shift operator on the Givental space). Let $X^{T}=\bigsqcup_{i} F_{i}$ be the decomposition of $X^{T}$ into connected components. Let $N_{i}$ be the normal bundle to $F_{i}$ in $X$. Let $N_{i}=\bigoplus_{\alpha} N_{i, \alpha}$ denote the $T$-eigenbundle decomposition, where $T$ acts on $N_{i, \alpha}$ by the character $\alpha \in \operatorname{Hom}\left(T, \mathbb{C}^{\times}\right)$. Let $\rho_{i, \alpha, j}, j=1, \ldots, \operatorname{rank}\left(N_{i, \alpha}\right)$ denote the Chern roots of $N_{i, \alpha}$. For a semi-negative $k \in \operatorname{Hom}\left(\mathbb{C}^{\times}, T\right)$, we define:

$$
\Delta_{i}(k)=Q^{\sigma_{i}-\sigma_{\min }} \prod_{\alpha} \prod_{j=1}^{\operatorname{rank}\left(N_{i, \alpha}\right)} \frac{\prod_{c=-\infty}^{0}\left(\rho_{i, \alpha, j}+\alpha+c z\right)}{\prod_{c=-\infty}^{-\alpha \cdot k}\left(\rho_{i, \alpha, j}+\alpha+c z\right)} \in H_{\widehat{T}}^{*}\left(F_{i}\right)_{\mathrm{loc}} \llbracket Q \rrbracket
$$

where $\alpha$ is regarded as an element of $H_{T}^{2}(\mathrm{pt}, \mathbb{Z}), \sigma_{i}$ is the section class of $E_{k}$ associated to a fixed point in $F_{i}$ and $\sigma_{\text {min }}$ is the minimal section class of $E_{k}$. Note that all but finite factors in the infinite product cancel. We define the operator $\mathcal{S}_{k}: H_{\widehat{T}}^{*}(X)_{\text {loc }} \rightarrow H_{\widehat{T}}^{*}(X)_{\text {loc }}$ by the following commutative diagram:

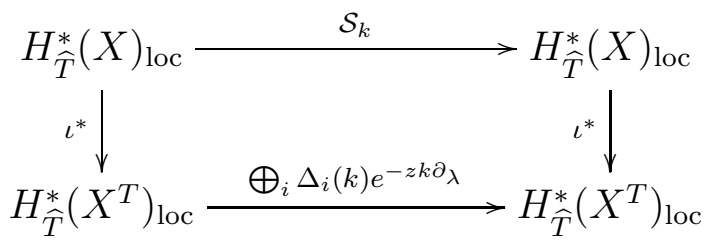

where we use the decomposition $H_{\widehat{T}}^{*}\left(X^{T}\right)_{\text {loc }} \cong \bigoplus_{i} H^{*}\left(F_{i}\right) \otimes \operatorname{Frac}\left(H_{\widehat{T}}(\mathrm{pt})\right)$ in the bottom arrow and $e^{-k z \partial_{\lambda}}$ acts on $\operatorname{Frac}\left(H_{\widehat{T}}(\mathrm{pt})\right)$ by $f(\lambda, z) \mapsto f(\lambda-k z, z)$. The operator $\mathcal{S}_{k}$ is a $(-k)$-twisted homomorphism.

The following is a key property of the shift operator.

Theorem 3.14. We have $M(\tau) \circ \mathbb{S}_{k}(\tau)=\mathcal{S}_{k} \circ M(\tau)$, where $M(\tau)$ is the fundamental solution in Proposition 2.9.

Proof. A similar intertwining property has been discussed in [OP10, BMO11, MO12]. We calculate $\widetilde{\mathbb{S}}_{k}(\tau)$ using $\widehat{T}$-equivariant localization. We refer the reader to GP99, CK99 for localization arguments in Gromov-Witten theory. Fix a section class $\hat{d} \in$ $\operatorname{Eff}\left(E_{k}\right)^{\text {sec }}$. A $\widehat{T}$-fixed stable map $f:\left(C, x_{1}, \ldots, x_{n+2}\right) \rightarrow E_{k}$ of degree $\hat{d}$ is of the form: 
- $C=C_{0} \cup C_{\text {sec }} \cup C_{\infty}$ with $C_{\text {sec }} \cong \mathbb{P}^{1}$;

- $f_{0}=\left.f\right|_{C_{0}}$ is a $T$-fixed stable map to $X_{0}$;

- $f_{\infty}=\left.f\right|_{C_{\infty}}$ is a $T$-fixed stable map to $X_{\infty}$;

- $f_{\mathrm{sec}}=\left.f\right|_{C_{\mathrm{sec}}}$ is a section of $E_{k}$ associated to a $T$-fixed point in $X$ (see (3.2)).

Recall that the tangent space $T^{1}$ and the obstruction space $T^{2}$ at the stable map $f$ fit into the exact sequence

$$
\begin{aligned}
& 0 \longrightarrow \operatorname{Ext}^{0}\left(\Omega_{C}^{1}(\mathbf{x}), \mathcal{O}_{C}\right) \longrightarrow H^{0}\left(C, f^{*} T_{E_{k}}\right) \longrightarrow T^{1} \\
& \longrightarrow \operatorname{Ext}^{1}\left(\Omega_{C}^{1}(\mathbf{x}), \mathcal{O}_{C}\right) \longrightarrow H^{1}\left(C, f^{*} T_{E_{k}}\right) \longrightarrow T^{2} \longrightarrow 0
\end{aligned}
$$

where $\mathbf{x}=x_{1}+\cdots+x_{n+2}$. The virtual normal bundle at $f$ is:

$$
\mathcal{N}^{\mathrm{vir}}=T^{1, \mathrm{mov}}-T^{2, \mathrm{mov}}=\chi\left(f^{*} T_{E_{k}}\right)^{\mathrm{mov}}-\chi\left(\Omega_{C}^{1}(\mathbf{x}), \mathcal{O}_{C}\right)^{\mathrm{mov}}
$$

where "mov" means the moving part with respect to the $\widehat{T}$-action and $\chi(\mathcal{E})=$ $H^{0}(C, \mathcal{E})-H^{1}(C, \mathcal{E}), \chi(\mathcal{E}, \mathcal{F})=\operatorname{Ext}^{0}(\mathcal{E}, \mathcal{F})-\operatorname{Ext}^{1}(\mathcal{E}, \mathcal{F})$ denotes the Euler characteristics. Let $p, q$ denote the nodal intersection points $C_{0} \cap C_{\text {sec }}, C_{\infty} \cap C_{\text {sec }}$ respectively. Using the normalization exact sequence $0 \rightarrow \mathcal{O}_{C} \rightarrow \mathcal{O}_{C_{0}} \oplus \mathcal{O}_{C_{\text {sec }}} \oplus \mathcal{O}_{C_{\infty}} \rightarrow \mathbb{C}_{p} \oplus \mathbb{C}_{q} \rightarrow 0$, we find:

$$
\begin{aligned}
\chi\left(f^{*} T_{E_{k}}\right)^{\mathrm{mov}} & =\chi\left(f_{0}^{*} T_{X_{0}}\right)^{\mathrm{mov}}+\chi\left(f_{\infty}^{*} T_{X_{\infty}}\right)^{\mathrm{mov}}+\chi\left(f_{\mathrm{sec}}^{*} T_{E_{k}}\right) \\
& +\xi+\xi^{-1}-\left(T_{f(p)} E\right)^{\mathrm{mov}}-\left(T_{f(q)} E\right)^{\mathrm{mov}}
\end{aligned}
$$

where $\xi$ is the one-dimensional $\mathbb{C}^{\times}$-representation of weight one. We write $\mathbf{x}=\mathbf{x}_{0}+\mathbf{x}_{\infty}$ where $\mathbf{x}_{0}, \mathbf{x}_{\infty}$ are divisors on $C_{0}, C_{\infty}$ respectively. Then we have

$$
\begin{aligned}
-\chi\left(\Omega_{C}^{1}(\mathbf{x}), \mathcal{O}_{C}\right)^{\mathrm{mov}} & =T_{p} C_{0} \otimes T_{p} C_{\mathrm{sec}}+T_{q} C_{\infty} \otimes T_{q} C_{\mathrm{sec}} \\
& -\chi\left(\Omega_{C_{0}}^{1}\left(\mathbf{x}_{0}+p\right), \mathcal{O}_{C_{0}}\right)^{\mathrm{mov}}-\chi\left(\Omega_{C_{\infty}}^{1}\left(\mathbf{x}_{\infty}+q\right), \mathcal{O}_{C_{\infty}}\right)^{\mathrm{mov}} .
\end{aligned}
$$

The $\widehat{T}$-fixed locus in the moduli space $\left(E_{k}\right)_{0, n+2, \hat{d}}$ is given by

$$
\bigsqcup_{i} \bigsqcup_{I_{1} \sqcup I_{2}=\{1, \ldots, n+2\}} \bigsqcup_{d_{0}+d_{\infty}+\sigma_{i}=\hat{d}}\left(\left(X_{0}\right)_{0, I_{1} \cup p, d_{0}}\right)^{T} \times_{F_{i}}\left(\left(X_{\infty}\right)_{0, I_{2} \cup q, d_{\infty}}\right)^{T}
$$

where $F_{i}, \sigma_{i}$ are as in Definition 3.13. Combining (3.4), (3.5), we find that the virtual normal bundle $\mathcal{N}_{i}^{\text {vir }}$ on the component $\left(\left(X_{0}\right)_{0, I_{1} \cup p, d_{0}}\right)^{T} \times_{F_{i}}\left(\left(X_{\infty}\right)_{0, I_{2} \cup q, d_{\infty}}\right)^{T}$ is:

$$
\mathcal{N}_{i}^{\mathrm{vir}}=\mathcal{N}_{0}^{\mathrm{vir}}+\mathcal{N}_{\infty}^{\mathrm{vir}}+\mathcal{N}_{\mathrm{sec}, i}-N_{F_{i} / X_{0}}-N_{F_{i} / X_{\infty}}+L_{p}^{-1} \otimes \xi+L_{q}^{-1} \otimes \xi^{-1}
$$

where $\mathcal{N}_{0}^{\text {vir }}$ is the virtual normal bundle of $\left(X_{0}\right)_{0, I_{1} \cup p, d_{0}}^{T}$ in $\left(X_{0}\right)_{0, I_{1} \cup p, d_{0}}, \mathcal{N}_{\infty}^{\text {vir }}$ is the virtual normal bundle of $\left(X_{\infty}\right)_{0, I_{2} \cup q, d_{\infty}}^{T}$ in $\left(X_{\infty}\right)_{0, I_{2} \cup q, d_{\infty}}, L_{p}$ (resp. $\left.L_{q}\right)$ is the universal cotangent line bundle at $p$ (resp. $q$ ) and $\mathcal{N}_{\text {sec }, i}$ is the vector bundle with fiber $\chi\left(f_{\mathrm{sec}}^{*} T_{E_{k}}\right)^{\text {mov }}$. Let $N_{F_{i} / X}=N_{i}=\oplus_{\alpha} N_{i, \alpha}$ be decomposition as in Definition 3.13. The normal bundle of $F_{i} \times \mathbb{P}^{1}$ in $E_{k}$ is

$$
\bigoplus_{\alpha} N_{i, \alpha} \otimes \mathcal{O}_{\mathbb{P}^{1}}(-\alpha \cdot k)
$$

Thus we find:

$$
\mathcal{N}_{\mathrm{sec}, i}=\xi \oplus \xi^{-1} \oplus \bigoplus_{\alpha} N_{i, \alpha} \otimes\left(\bigoplus_{c \leq 0} \xi^{c}-\bigoplus_{c<\alpha \cdot k} \xi^{c}\right)
$$


The virtual localization formula gives:

$$
\begin{aligned}
\left(\widetilde{\mathbb{S}}_{k}(\tau) \alpha, \beta\right)= & \sum_{i, k, l, a, b} \sum_{d_{0}+d_{\infty}+\sigma_{i}=\hat{d}}\left\langle z \alpha, \tau, \ldots, \tau, \frac{\left(\iota_{0, i}\right)_{*} \phi_{i, a}}{z-\psi}\right\rangle_{0, k+2, d_{0}}^{X_{0}, \widehat{T}} \frac{Q^{d_{0}}}{k !} \\
& \times\left(\int_{F_{i}} \frac{Q^{\sigma_{i}-\sigma_{\min }}}{e_{\widehat{T}}\left(\mathcal{N}_{\mathrm{sec}, i}\right)} \phi_{i}^{a} \phi_{i}^{b}\right)\left\langle\frac{\left(\iota_{\infty, i}\right)_{*} \phi_{i, b}}{-z-\psi}, \tau^{\prime}, \ldots, \tau^{\prime},-z \beta\right\rangle_{0, l+2, d_{\infty}}^{X_{\infty}, \widehat{T}} \frac{Q^{d_{\infty}}}{l !}
\end{aligned}
$$

where $\alpha \in H_{\widehat{T}}^{*}\left(X_{0}\right), \beta \in H_{\widehat{T}}^{*}\left(X_{\infty}\right), \tau^{\prime}=\Phi_{k}(\tau)$, the maps $\iota_{0, i}: F_{i} \rightarrow X_{0}, \iota_{\infty, i}: F_{i} \rightarrow X_{\infty}$ are the natural inclusions, $\left\{\phi_{i, a}\right\} \subset H^{*}\left(F_{i}\right)$ is a basis, $\left\{\phi_{i}^{a}\right\}$ is the dual basis such that $\int_{F_{i}} \phi_{i, a} \cup \phi_{i}^{b}=\delta_{a}^{b}$. Note that we have by (3.6),

$$
\frac{Q^{\sigma_{i}-\sigma_{\min }}}{e_{\widehat{T}}\left(\mathcal{N}_{\mathrm{vir}, i}\right)}=\frac{1}{z(-z)} \frac{1}{e_{\widehat{T}}\left(N_{F_{i} / X_{\infty}}\right)}\left(e^{k z \partial_{\lambda}} \Delta_{i}(k)\right) .
$$

Combining these equations, we conclude

$$
\left(\widetilde{\mathbb{S}}_{k}(\tau) \alpha, \beta\right)=\left(\widetilde{\mathcal{S}}_{k} M(\tau, z) \alpha, M^{\prime}\left(\tau^{\prime},-z\right) \beta\right)
$$

where we write the argument $z$ in the fundamental solution explicitly and

- $\widetilde{\mathcal{S}}_{k}: H_{\widehat{T}}\left(X_{0}\right)_{\text {loc }} \rightarrow H_{\widehat{T}}\left(X_{\infty}\right)_{\text {loc }}$ is a map defined similarly to $\mathcal{S}_{k}$ by replacing $\bigoplus_{i} \Delta_{i}(k) e^{-k z \partial_{\lambda}}$ in the diagram (3.3) with $\bigoplus_{i}\left(e^{k z \partial_{\lambda}} \Delta_{i}(k)\right)$;

- $M^{\prime}\left(\tau^{\prime}, z\right)$ is defined similarly to Proposition 2.2 by replacing $T$-equivariant Gromov-Witten invariants there with $\left(\widehat{T}, \rho_{k}\right)$-equivariant invariants.

Note that $M^{\prime}\left(\tau^{\prime}, z\right)=\Phi_{k} \circ M(\tau, z) \circ \Phi_{k}^{-1}$ and $\widetilde{\mathcal{S}}=\Phi_{k} \circ \mathcal{S}$. The conclusion follows from the so-called "unitarity" $M(\tau,-z)^{*}=M(\tau, z)^{-1}$ of the fundamental solution (see Giv98a, §1]).

Theorem 3.14 and the differential equation $\partial_{i} \circ M(\tau)=M(\tau) \circ \nabla_{i}$ show:

Corollary 3.15. The shift operator commutes with the quantum connection, i.e. $\left[\nabla_{i}, \mathbb{S}_{k}(\tau)\right]=0$ for $i=0, \ldots, N$.

This corollary is shown in MO12, §8] in the case where $\tau=0$. We also remark that the shift operators commute each other.

Corollary 3.16. We have $\mathcal{S}_{k} \circ \mathcal{S}_{l}=Q^{d(k, l)} \mathcal{S}_{k+l}$ for some $d(k, l) \in H_{2}(X, \mathbb{Z})$ which is symmetric in $k$ and l. In particular, $\mathbb{S}_{k} \circ \mathbb{S}_{l}=Q^{d(k, l)} \mathbb{S}_{k+l},\left[\mathcal{S}_{k}, \mathcal{S}_{l}\right]=\left[\mathbb{S}_{k}, \mathbb{S}_{l}\right]=0$.

Proof. Consider the $X$-bundle $E_{k, l}$ over $\mathbb{P}^{1} \times \mathbb{P}^{1}$ given by

$$
E_{k, l}=X \times\left(\mathbb{C}^{2} \backslash\{0\}\right) \times\left(\mathbb{C}^{2} \backslash\{0\}\right) / \mathbb{C}^{\times} \times \mathbb{C}^{\times}
$$

where $\left(s_{1}, s_{2}\right) \in \mathbb{C}^{\times} \times \mathbb{C}^{\times}$acts on $X \times \mathbb{C}^{2} \times \mathbb{C}^{2}$ by $\left(s_{1}, s_{2}\right) \cdot\left(x,\left(a_{1}, a_{2}\right),\left(b_{1}, b_{2}\right)\right)=$ $\left(s_{1}^{k} s_{2}^{l},\left(s_{1}^{-1} a_{1}, s_{1}^{-1} a_{2}\right),\left(s_{2}^{-1} b_{1}, s_{2}^{-1} b_{2}\right)\right)$. Note that $\left.E_{k, l}\right|_{\mathbb{P}^{1} \times[1: 0]} \cong E_{k}$ and $\left.E_{k, l}\right|_{[1: 0] \times \mathbb{P}^{1}} \cong E_{l}$ and $\left.E_{k, l}\right|_{\Delta\left(\mathbb{P}^{1}\right)} \cong E_{k+l}$, where $\Delta\left(\mathbb{P}^{1}\right) \subset \mathbb{P}^{1} \times \mathbb{P}^{1}$ denotes the diagonal. The addition in $H_{2}\left(E_{k, l}, \mathbb{Z}\right)$ defines a map $\#: H_{2}^{\sec }\left(E_{l}, \mathbb{Z}\right) \times H_{2}^{\sec }\left(E_{k}, \mathbb{Z}\right) \rightarrow H_{2}^{\sec }\left(E_{k+l}, \mathbb{Z}\right)$. For any $T$-fixed point $x$, the section class $\sigma_{x}$ (see (3.2)) associated to $x$ satisfies $\sigma_{x} \# \sigma_{x}=\sigma_{x}$. A straightforward computation now shows that $\mathcal{S}_{k} \circ \mathcal{S}_{l}=Q^{\sigma_{\min }(k+l)-\sigma_{\min }(k) \# \sigma_{\min }(l)} \mathcal{S}_{k+l}$, 
where $\sigma_{\min }(k)$ denotes the minimal section class of $E_{k}$. The conclusion follows by setting $d(k, l)=\sigma_{\min }(k+l)-\sigma_{\min }(k) \# \sigma_{\min }(l)$ and the commutativity of \#.

3.4. Relation to the Seidel Representation. Taking the $z \rightarrow 0$ limit of shift operators, we obtain a big quantum cohomology version of the Seidel representation [Sei97]. The author learned the idea of big Seidel elements from Eduardo Gonzalez during joint work GI12 with him.

Definition 3.17 (Seidel elements). Let $k \in \operatorname{Hom}\left(\mathbb{C}^{\times}, T\right)$ be a semi-negative homomorphism. The element $S_{k}(\tau):=\lim _{z \rightarrow 0} \mathbb{S}_{k}(\tau) 1$ of $H_{T}^{*}(X) \llbracket Q \rrbracket \llbracket \tau^{0}, \ldots, \tau^{m} \rrbracket$ is called the Seidel element.

By Corollary 3.15, the $z \rightarrow 0$ limit of the operator $\mathbb{S}_{k}(\tau)$ commutes with the quantum multiplication, and therefore coincides with the quantum multiplication by $S_{k}(\tau)$ (see also [MO12, §8]). By Corollary 3.16, we have

$$
S_{k}(\tau) \star S_{l}(\tau)=Q^{d(k, l)} S_{k+l}(\tau) .
$$

This is called the Seidel representation.

3.5. Relation to the $\widehat{\Gamma}$-Integral Structure. We remark a relationship between the shift operator and the $\widehat{\Gamma}$-integral structure introduced in [ri09, KKP08, CIJ14. For quantum cohomology of the Hilbert scheme of points on $\mathbb{C}^{2}$, it has been observed in OP10 that certain $\Gamma$-factors play an important role in the difference equation associated to the shift operators.

We recall the $\widehat{\Gamma}$-class of $X$. Let $\delta_{1}, \ldots, \delta_{D}$ denote the $T$-equivariant Chern roots of the tangent bundle $T X$ such that $c^{T}(T X)=\left(1+\delta_{1}\right) \cdots\left(1+\delta_{D}\right)$. The $T$-equivariant $\widehat{\Gamma}$-class of $X$ is the class

$$
\widehat{\Gamma}_{X}=\widehat{\Gamma}(T X)=\prod_{i=1}^{D} \Gamma\left(1+\delta_{i}\right)
$$

in $H_{T}^{* *}(X)=\prod_{p=0}^{\infty} H_{T}^{p}(X)$. Here $\Gamma(z)=\int_{0}^{\infty} e^{-t} t^{z-1} d t$ is Euler's $\Gamma$-function. By Taylor expansion, the right-hand side becomes a symmetric formal power series in $\delta_{1}, \ldots, \delta_{D}$ and thus can be expressed in terms of the equivariant Chern classes of $T X$.

The $\widehat{\Gamma}$-integral structure assigns the following homogeneous flat section $\mathfrak{s}(E)$ of the quantum connection to a $T$-equivariant vector bundle $E \rightarrow X$ :

$$
\mathfrak{s}(E)=(2 \pi)^{-D / 2} M(\tau)^{-1} z^{-\mu} z^{c_{1}(X)} \widehat{\Gamma}_{X}(2 \pi i)^{\operatorname{deg} / 2} \operatorname{ch}^{T}(E)
$$

where $D=\operatorname{dim}_{\mathbb{C}} X, M(\tau)$ is the fundamental solution in Proposition 2.2, $\mu \in$ $\operatorname{End}_{\mathbb{C}}\left(H_{T}^{*}(X)\right)$ is the Hodge grading operator defined by $\mu\left(\phi_{i}\right)=\left(\frac{\operatorname{deg} \phi_{i}}{2}-\frac{D}{2}\right) \phi_{i}$, $z^{c_{1}(X)}=e^{c_{1}(X) \log z}$ and $(2 \pi i)^{\operatorname{deg} / 2} \operatorname{ch}^{T}(E)=\sum_{p=0}^{\infty}(2 \pi i)^{p} \operatorname{ch}_{p}^{T}(E)$. The section $\mathfrak{s}(E)$ is flat, i.e. $\nabla_{i} \mathfrak{s}(E)=0$ and is homogeneous in the sense that

$$
\left[z \frac{\partial}{\partial z}+\mu+\sum_{i=0}^{N}\left(1-\frac{1}{2} \operatorname{deg} \phi_{i}\right) \tau^{i} \frac{\partial}{\partial \tau^{i}}+\sum_{i=0}^{N} \rho^{i} \frac{\partial}{\partial \tau^{i}}\right] \mathfrak{s}(E)=0
$$

where we set $c_{1}(X)=\sum_{i=0}^{N} \rho^{i} \phi_{i}$. A key property of $\mathfrak{s}(E)$ is that the pairing

$$
\left(\mathfrak{s}(E)\left(\tau, e^{-\pi \dot{i}} z\right), \mathfrak{s}(F)(\tau, z)\right)
$$


equals the $T$-equivariant Euler pairing $z^{-\operatorname{deg} / 2}(2 \pi i)^{\operatorname{deg} / 2} \chi(E, F)$, where $\chi(E, F)=$ $\sum_{i=0}^{D}(-1)^{i} \operatorname{ch}^{T}\left(\operatorname{Ext}^{i}(E, F)\right) \in H_{T}^{* *}(\mathrm{pt})$. This follows from an appropriate equivariant Hirzebruch-Riemann-Roch formula. See [C1J14, §2-3] for more details.

The $T$-equivariant $K$-group is a module over $K_{T}^{0}(\mathrm{pt})=\mathbb{C}[T]$ and the Chern character $\mathrm{ch}^{T}: K_{T}^{0}(\mathrm{pt}) \rightarrow H_{T}^{* *}(\mathrm{pt})$ can be viewed as the pull-back by the universal covering exp: $\operatorname{Lie}(T)=\mathbb{C}^{m} \rightarrow T=\left(\mathbb{C}^{\times}\right)^{m}$. A deck-transformation of this covering is given by the shift] of equivariant parameters $\lambda_{j} \rightarrow \lambda_{j}+2 \pi i$. This suggests that $\mathfrak{s}(E)$ should be "invariant" under integral shifts of equivariant parameters.

Proposition 3.18. When the Novikov variable $Q$ is set to be one, the flat section $\mathfrak{s}(E)$ is invariant under the shift operator:

$$
\mathbb{S}_{k} \mathfrak{s}(E)=\mathfrak{s}(E)
$$

for every semi-negative $k \in \operatorname{Hom}\left(\mathbb{C}^{\times}, T\right)$.

Proof. As is discussed in CIJ14, §3], the divisor equation shows that the specialization $Q=1$ of the Novikov variable is well-defined for $\mathfrak{s}(E)$. In view of the intertwining property in Theorem 3.14, it suffices to show that

$$
\widetilde{\mathcal{S}}_{k}\left(z^{-\mu} z^{c_{1}(X)} \widehat{\Gamma}_{X}(2 \pi i)^{\operatorname{deg} / 2} \operatorname{ch}(E)\right)=z^{-\mu} z^{c_{1}(X)} \widehat{\Gamma}_{X}(2 \pi i)^{\operatorname{deg} / 2} \operatorname{ch}(E) .
$$

The restriction to the $T$-fixed component $F_{i}$ gives

$$
\begin{aligned}
{\left[z^{-\mu} z^{c_{1}(X)} \widehat{\Gamma}_{X}(2 \pi i)^{\operatorname{deg} / 2} \operatorname{ch}(E)\right]_{F_{i}} } & =z^{D / 2} z^{c_{1}\left(F_{i}\right) / z}\left(z^{-\operatorname{deg} / 2} \widehat{\Gamma}_{F_{i}}\right) \\
\times & \left(\prod_{\alpha} \prod_{j=1}^{\operatorname{rank} N_{\alpha, i}} z^{\left(\rho_{i, \alpha, j}+\alpha\right) / z} \Gamma\left(1+\frac{\rho_{i, \alpha, j}}{z}+\frac{\alpha}{z}\right)\right) \sum_{\epsilon} e^{2 \pi i \epsilon / z}
\end{aligned}
$$

where $\epsilon$ ranges over $T$-equivariant Chern roots of $E$ and we use the notation from Definition 3.13. The conclusion easily follows from the identity $\Gamma(1+z)=z \Gamma(z)$.

\section{Toric Mirror TheOrem}

In this section we give a new proof of a mirror theorem Giv98b for toric manifolds.

4.1. Toric Manifolds. We fix notation for toric manifolds. For background materials on toric manifolds, we refer the reader to Oda88, Aud04, CLS11. Let $\mathbf{N} \cong \mathbb{Z}^{D}$ denote a lattice. A toric manifold is given by a rational simplicial fan $\Sigma$ in the vector space $\mathbf{N}_{\mathbb{R}}=\mathbf{N} \otimes \mathbb{R}$. We assume that

- each cone $\sigma$ of $\Sigma$ is generated by part of a $\mathbb{Z}$-basis of $\mathbf{N}$;

- the support $|\Sigma|=\bigcup_{\sigma \in \Sigma} \sigma$ of $\Sigma$ is convex and full-dimensional;

- $\Sigma$ admits a strictly convex piecewise linear function $\eta:|\Sigma| \rightarrow \mathbb{R}$.

These assumptions ensure that the corresponding toric variety $X_{\Sigma}$ is smooth and satisfies the hypotheses in $\$ 2.1$. We do not require that $X$ is compact, or $c_{1}(X)$ is semipositive. Let $b_{1}, \ldots, b_{m} \in \mathbf{N}$ be primitive integral generators of one-dimensional cones of

\footnotetext{
${ }^{2}$ the shift by $2 \pi i$ is superseded by the shift by $z$ because of the operators $z^{-\mu}$ and $(2 \pi i)^{\operatorname{deg} / 2}$.
} 
$\Sigma$. Let $\beta: \mathbb{Z}^{m} \rightarrow \mathbf{N}$ be the homomorphism sending the standard basis vector $e_{i} \in \mathbb{Z}^{m}$ to $b_{i}$. The fan sequence is the exact sequence

$$
0 \longrightarrow \mathbb{L} \longrightarrow \mathbb{Z}^{m} \stackrel{\beta}{\longrightarrow} \mathbf{N} \longrightarrow 0
$$

with $\mathbb{L}=\operatorname{Ker}(\beta)$. Set $K=\mathbb{L} \otimes \mathbb{C}^{\times}$. The inclusion $\mathbb{L} \hookrightarrow \mathbb{Z}^{m}$ induces the inclusion $K \hookrightarrow\left(\mathbb{C}^{\times}\right)^{m}$ of tori and defines a linear $K$-action on $\mathbb{C}^{m}$. The toric variety associated to $\Sigma$ is given by the GIT quotient

$$
X_{\Sigma}=U / K, \quad U=\mathbb{C}^{m} \backslash Z
$$

where $Z \subset \mathbb{C}^{m}$ is the common zero set of monomials $z^{I}=z_{i_{1}} \cdots z_{i_{k}}$ with $I=\left\{i_{1}, \ldots, i_{k}\right\}$ such that $\left\{b_{i}: 1 \leq i \leq m, i \notin I\right\}$ spans a cone in $\Sigma$. We consider the $T$-action on $X_{\Sigma}$ indueced by the $T=\left(\mathbb{C}^{\times}\right)^{m}$-action on $\mathbb{C}^{m}$.

Let $\lambda_{i} \in H_{T}^{2}(\mathrm{pt}) \cong \operatorname{Lie}(T)^{*}$ denote the class corresponding to the $i$ th projection $T \rightarrow \mathbb{C}^{\times}$. We have

$$
H_{T}^{*}(\mathrm{pt})=\mathbb{C}\left[\lambda_{1}, \ldots, \lambda_{m}\right]
$$

All the $T$-weights of $H^{0}\left(X_{\Sigma}, \mathcal{O}\right)$ are contained in the cone $\sum_{i=1}^{m} \mathbb{R}_{\geq 0}\left(-\lambda_{i}\right)$ and therefore the condition (2) in $\S 2.1$ is satisfied. A cocharacter $k: \mathbb{C}^{\times} \rightarrow T$ is semi-negative in the sense of Definition 3.3 if $\lambda_{i} \cdot k \geq 0$ for all $i=1, \ldots, m$.

Let $u_{i} \in H_{T}^{2}\left(X_{\Sigma}\right)$ denote the class of the torus-invariant divisor $\left\{z_{i}=0\right\}$ defined as the vanishing set of the $i$ th co-ordinate $z_{i}$ on $\mathbb{C}^{m}$. The $T$-equivariant cohomology ring of $X_{\Sigma}$ is generated by these classes:

$$
H_{T}^{*}\left(X_{\Sigma}\right) \cong H_{T}^{*}(\mathrm{pt})\left[u_{1}, \ldots, u_{m}\right] /\left(\mathfrak{I}_{1}+\mathfrak{I}_{2}\right)
$$

where $\mathfrak{I}_{1}$ is the ideal generated by $\prod_{i \in I} u_{i}$ such that $\left\{b_{i}: i \in I\right\}$ does not span a cone in $\Sigma$ and $\mathfrak{I}_{2}$ is the ideal generated by $\sum_{i=1}^{m} \chi\left(b_{i}\right)\left(u_{i}-\lambda_{i}\right)$ with $\chi \in \operatorname{Hom}(\mathbf{N}, \mathbb{Z})$.

4.2. Mirror Theorem. Define a cohomology-valued hypergeometric series $I(y, z)$ by the formula:

$$
I(y, z)=z e^{\sum_{i=1}^{m} u_{i} \log y_{i} / z} \sum_{d \in \operatorname{Eff}\left(X_{\Sigma}\right)}\left(\prod_{i=1}^{m} \frac{\prod_{c=-\infty}^{0}\left(u_{i}+c z\right)}{\prod_{c=-\infty}^{u_{i} \cdot d}\left(u_{i}+c z\right)}\right) Q^{d} y_{1}^{u_{1} \cdot d} \cdots y_{m}^{u_{m} \cdot d} .
$$

This formula defines an element of $H_{\widehat{T}}^{*}\left(X_{\Sigma}\right)_{\operatorname{loc}} \llbracket Q \rrbracket \llbracket \log y \rrbracket$. We may write $I(y, z)$ as a sum over $H_{2}\left(X_{\Sigma}, \mathbb{Z}\right)$ since the summand automatically vanishes if $d \notin \mathrm{Eff}\left(X_{\Sigma}\right)$.

Givental's mirror theorem Giv98b (generalized later in [LLY99, ¿ri08, Bro09) states the following:

Theorem 4.1. The function $I(y,-z)$ lies on the Givental cone associated to genus-zero Gromov-Witten theory of $X_{\Sigma}$.

We explain the meaning of the statement. The Givental cone $\mathcal{L}$ Giv04 is a subset of $H_{\widehat{T}}^{*}\left(X_{\Sigma}\right)_{\text {loc }} \llbracket Q \rrbracket$ consisting of points of the form:

$$
-z+\mathbf{t}(z)+\sum_{i=0}^{N} \sum_{n=0}^{\infty} \sum_{d \in \operatorname{Eff}\left(X_{\Sigma}\right)} \frac{Q^{d}}{n !}\left\langle\frac{\phi^{i}}{-z-\psi}, \mathbf{t}(\psi), \ldots, \mathbf{t}(\psi)\right\rangle_{0, n+1, d}^{X, T} \phi_{i}
$$

with $\mathbf{t}(z) \in H_{\widehat{T}}^{*}\left(X_{\Sigma}\right) \llbracket Q \rrbracket=H_{T}^{*}\left(X_{\Sigma}\right)[z] \llbracket Q \rrbracket$. The Givental cone $\mathcal{L}$ can be written as the graph of the differential of the genus-zero descendant Gromov-Witten potential, and 
encodes all genus-zero descendant Gromov-Witten invariants. Theorem 4.1 says that $I(y, z)$ is of the form (4.1) for some $\mathbf{t}(z) \in H_{T}^{*}\left(X_{\Sigma}\right)[z] \llbracket Q \rrbracket \llbracket \log y \rrbracket$ with $\left.\mathbf{t}(z)\right|_{Q=\log y=0}=0$. For toric manifolds, the above $I$-function determines the Givental cone and hence all the genus-zero Gromov-Witten invariants completely.

In this paper, we use an alternative description [Giv04 of the Givental cone $\mathcal{L}$. We can write $\mathcal{L}$ as the union

$$
\mathcal{L}=\bigcup_{\tau \in H_{T}^{*}\left(X_{\Sigma}\right) \llbracket Q \rrbracket} z T_{\tau}
$$

of the semi-infinite subspaces $T_{\tau}=M(\tau,-z) H_{T}\left(X_{\Sigma}\right)[z] \llbracket Q \rrbracket$, where $M(\tau,-z)$ denotes the fundamental solution from Proposition 2.2 with the sign of $z$ flipped. The subspace $T_{\tau}$ is a (common) tangent space to $\mathcal{L}$ along $z T_{\tau} \subset \mathcal{L}$. Therefore, it suffices to show that $I(y, z)$ can be written in the form

$$
I(y, z)=z M(\tau(y), z) \Upsilon(y, z)
$$

for some $\tau(y) \in H_{T}^{*}\left(X_{\Sigma}\right) \llbracket Q \rrbracket \llbracket \log y \rrbracket$ and $\Upsilon(y, z) \in H_{T}^{*}\left(X_{\Sigma}\right)[z] \llbracket Q \rrbracket \llbracket \log y \rrbracket$.

4.3. Proof. The idea of the proof is as follows. Let $e_{i}$ denote the cocharacter $\mathbb{C}^{\times} \rightarrow$ $T=\left(\mathbb{C}^{\times}\right)^{m}$ given by the inclusion of the $i$ th factor. Let $\mathbb{S}_{i}=\mathbb{S}_{e_{i}}, \mathcal{S}_{i}=\mathcal{S}_{e_{i}}$ denote the corresponding shift operators. In view of Theorem 3.14, the shift operator $\mathcal{S}_{i}$ defines a vector field on the Givental cone:

$$
\mathcal{L} \ni \mathbf{f} \longmapsto z^{-1} \mathcal{S}_{i} \mathbf{f} \in T_{\mathbf{f}} \mathcal{L}
$$

These vector fields define commuting flows by Corollary 3.16. We will identify the $I$-function with an integral submanifold of these vector fields.

Consider the $\mathbb{C}^{\times}$-action on $X_{\Sigma}$ induced by the cocharacter $e_{i} \in \operatorname{Hom}\left(\mathbb{C}^{\times}, T\right)$. The minimal fixed component $F_{\min }$ for this $\mathbb{C}^{\times}$-action is the toric divisor $\left\{z_{i}=0\right\}$. Let $E_{i}=E_{e_{i}}$ denote the associated bundle. For a fixed point $x \in X_{\Sigma}^{T}$, we set $d_{i}(x)=$ $\sigma_{x}-\sigma_{\min } \in H_{2}\left(X_{\Sigma}, \mathbb{Z}\right)$, where $\sigma_{x} \in H_{2}^{\sec }\left(E_{k}\right)$ is the section (3.2) of $E_{i}$ associated to $x$ and $\sigma_{\min } \in H_{2}^{\mathrm{sec}}\left(E_{k}\right)$ is the minimal section class of $E_{i}$. We write $u_{j}(x) \in H_{T}^{2}(\mathrm{pt})$ for the restriction of $u_{j}$ to $x$.

Lemma 4.2. With the notation as above, we have

$$
u_{j}(x) \cdot e_{i}=\delta_{i j}-u_{j} \cdot d_{i}(x) .
$$

Proof. Consider the $\widehat{T}$-invariant divisor $\left\{z_{j}=0\right\} \times \mathbb{P}^{1}$ in $E_{i}$ and let $\hat{u}_{j}$ denote the $\widehat{T}$-equivariant Poincaré dual of the divisor. Then we have $\left.\hat{u}_{j}\right|_{(x,[1,0])}=u_{j}(x)$ and $\left.\hat{u}_{j}\right|_{(x,[0,1])}=u_{j}(x)+\left(u_{j}(x) \cdot e_{i}\right) z$. The localization formula gives

$$
\hat{u}_{j} \cdot \sigma_{x}=\frac{\left.\hat{u}_{j}\right|_{(x,[1,0])}}{z}+\frac{\left.\hat{u}_{j}\right|_{(x,[0,1])}}{-z}=-u_{j}(x) \cdot e_{i} .
$$

Similarly we have $\hat{u}_{j} \cdot \sigma_{\min }=-u_{j}(y) \cdot e_{i}$ for any $T$-fixed point $y$ in the divisor $F_{\min }=$ $\left\{z_{i}=0\right\}$. If $i \neq j$, taking $y$ away from $\left\{z_{j}=0\right\}$, we get $u_{j}(y)=0$. If $i=j, u_{j}(y) \cdot e_{i}=1$. Therefore $\hat{u}_{j} \cdot \sigma_{\min }=-\delta_{i j}$. The conclusion follows. 
Lemma 4.3. The I-function is an integral curve of the vector field (4.2), that is, for $i \in\{1, \ldots, m\}$, we have

$$
z \frac{\partial}{\partial y_{i}} I(y, z)=\mathcal{S}_{i} I(y, z)
$$

Proof. Note that all the $T$-fixed points on $X_{\Sigma}$ are isolated. Let $x \in X^{T}$ be a fixed point. It suffices to show that

$$
z \frac{\partial}{\partial y_{i}} I_{x}(y, z)=\Delta_{x}\left(e_{i}\right) e^{-z \partial_{\lambda_{i}}} I_{x}(y, z)
$$

where $I_{x}(y, z)$ is the restriction of the $I$-function to $x$ and

$$
\Delta_{x}\left(e_{i}\right)=Q^{d_{i}(x)} \prod_{j=1}^{m} \frac{\prod_{c=-\infty}^{0}\left(u_{j}(x)+c z\right)}{\prod_{c=-\infty}^{-u_{j}(x) \cdot e_{i}}\left(u_{j}(x)+c z\right)} .
$$

Using Lemma 4.2, we have

$$
\begin{aligned}
& \Delta_{x}\left(e_{i}\right) e^{-z \partial_{\lambda_{i}}} I_{x}(y, z)=z e^{\sum_{j=1}^{m} u_{j}(x) \log y_{j} / z} e^{-\log y_{i}+\sum_{j=1}^{m}\left(u_{j} \cdot d_{i}(x)\right) \log y_{j}} \\
& \quad \times Q^{d_{i}(x)} \sum_{d \in H_{2}\left(X_{\Sigma}, \mathbb{Z}\right)}\left(\prod_{j=1}^{m} \frac{\prod_{c=-\infty}^{0}\left(u_{j}(x)+c z\right)}{\prod_{c=-\infty}^{-u_{j}(x) \cdot e_{i}}\left(u_{j}(x)+c z\right)} \frac{\prod_{c=-\infty}^{-u_{j}(x) \cdot e_{i}}\left(u_{j}(x)+c z\right)}{\prod_{c=-\infty}^{u_{j} \cdot d-u_{j}(x) \cdot e_{i}}\left(u_{j}(x)+c z\right)}\right) Q^{d} y^{d}
\end{aligned}
$$

where $y^{d}=\prod_{j=1}^{m} y_{j}^{u_{j} \cdot d}$. Changing variables $d \rightarrow d-d_{i}(x)$ and using again Lemma 4.2, we find that this equals $z \frac{\partial}{\partial y_{i}} I(y, z)$.

We identify the classical shift operators:

Notation 4.4. We set $v_{i}:=u_{i}-\lambda_{i} \in H_{T}^{2}\left(X_{\Sigma}\right)$ and write $v_{i}(x) \in H_{T}^{2}(\mathrm{pt})$ for the restriction of $v_{i}$ to a $T$-fixed point $x$.

Lemma 4.5. Let $f(v, \lambda)$ be a cohomology class in $H_{T}^{*}\left(X_{\Sigma}\right)$ expressed as a polynomial in $v_{1}, \ldots, v_{m}$ and $\lambda_{1}, \ldots, \lambda_{m}$. When we write $\tau \in H_{T}^{*}\left(X_{\Sigma}\right)$ as a polynomial $\tau(v, \lambda)$ in $v_{1}, \ldots, v_{m}$ and $\lambda_{1}, \ldots, \lambda_{m}$, we have

$$
\lim _{Q \rightarrow 0} \mathbb{S}_{i}(\tau) f(v, \lambda)=u_{i} e^{\left(\tau\left(v, \lambda-e_{i} z\right)-\tau(v, \lambda)\right) / z} f\left(v, \lambda-z e_{i}\right)
$$

where $\lambda-z e_{i}=\left(\lambda_{1}, \ldots, \lambda_{i-1}, \lambda_{i}-z, \lambda_{i+1}, \ldots, \lambda_{m}\right)$. In particular the classical Seidel elements are given by:

$$
\lim _{Q \rightarrow 0} S_{i}(\tau)=u_{i} e^{-\frac{\partial \tau(v, \lambda)}{\partial \lambda_{i}}}
$$

Proof. Recall from Theorem 3.14 that we have $\mathcal{S}_{i} \circ M(\tau)=M(\tau) \circ \mathbb{S}_{i}(\tau)$. Since $\lim _{Q \rightarrow 0} M(\tau)=e^{\tau / z}$, we have

$$
\lim _{Q \rightarrow 0} \mathbb{S}_{i}(\tau) f(v, \lambda)=e^{-\tau / z}\left(\lim _{Q \rightarrow 0} \mathcal{S}_{i}\right) e^{\tau / z} f(v, \lambda) .
$$

By definition of $\mathcal{S}_{i}$, this vanishes when restricted to a fixed point outside of the minimal fixed component $\left\{z_{i}=0\right\}$ with respect to $e_{i}$. On the other hand, for any $T$-fixed point 
$x$ in $\left\{z_{i}=0\right\}$, Lemma 4.2 implies that $u_{j}(x) \cdot e_{i}=\delta_{i j}, v_{j}(x) \cdot e_{i}=0$ and thus:

$$
\begin{aligned}
\left.\lim _{Q \rightarrow 0} \mathbb{S}_{i}(\tau) f(v, \lambda)\right|_{x} & =e^{-\tau(v(x), \lambda) / z} u_{i}(x) e^{-z \partial_{\lambda_{i}}}\left[e^{\tau(v(x), \lambda) / z} f(v(x), \lambda)\right] \\
& =u_{i}(x) e^{\left(\tau\left(v(x), \lambda-e_{i} z\right)-\tau(v(x), \lambda)\right) / z} f\left(v(x), \lambda-e_{i} z\right)
\end{aligned}
$$

where we set $v(x)=\left(v_{1}(x), \ldots, v_{m}(x)\right)$. The conclusion follows.

Lemma 4.6. Let $x$ be a $T$-fixed point on $X_{\Sigma}$. The restriction $u_{j}(x)$ is a linear combination of $\lambda_{i}$ such that $x$ does not lie on the divisor $\left\{z_{i}=0\right\}$.

Proof. Note that if $x$ does not lie on the divisor $\left\{z_{i}=0\right\}$, we have $u_{i}(x)=0$ and thus $v_{i}(x)=-\lambda_{i}$. This together with the linear relation $\sum_{i=1}^{m} \chi\left(b_{i}\right) v_{i}=0, \chi \in \operatorname{Hom}(\mathbf{N}, \mathbb{Z})$ determines $v_{1}(x), \ldots, v_{m}(x)$ uniquely. This implies the conclusion.

Let $\overline{\mathcal{L}}=\left.\mathcal{L}\right|_{z \rightarrow-z}$ denote the Givental cone with the sign of $z$ flipped. By the description in $\$ 4.2$, we have a parametrization of the Givental cone $\overline{\mathcal{L}}$ by $(\tau, \Upsilon) \in$ $H_{T}^{*}(X) \times H_{\widehat{T}}^{*}(X)=H_{T}^{*}(X) \times H_{T}^{*}(X)[z]$ as:

$$
(\tau, \Upsilon) \longmapsto z M(\tau, z) \Upsilon \in \overline{\mathcal{L}}
$$

The vector field (4.2) on $\overline{\mathcal{L}}$ corresponds to the following vector field on $H_{T}^{*}(X) \times$ $H_{T}^{*}(X)[z]$ :

$$
\left(\mathbf{V}_{i}\right)_{\tau, \Upsilon}=\left(S_{i}(\tau),\left[z^{-1} \mathbb{S}_{i}(\tau)\right]_{+} \Upsilon\right)
$$

where $S_{i}(\tau)$ is the Seidel element in Definition 3.17 and $[\cdots]_{+}$means the projection to the polynomial part in $z$, i.e. $\left[z^{-1} \mathbb{S}_{i}(\tau)\right]_{+} \Upsilon=z^{-1} \mathbb{S}_{i}(\tau) \Upsilon-z^{-1} S_{i}(\tau) \star_{\tau} \Upsilon$. In fact, if we have a curve $t \mapsto(\tau(t), \Upsilon(t))$ with $\tau^{\prime}(0)=S_{i}(\tau(0)), \Upsilon^{\prime}(0)=\left[z^{-1} \mathbb{S}_{i}(\tau(0))\right]_{+} \Upsilon(0)$, the corresponding curve $\mathbf{f}(t)=z M(\tau(t), z) \Upsilon(t)$ on $\overline{\mathcal{L}}$ satisfies

$$
\begin{aligned}
\mathbf{f}^{\prime}(0) & =M(\tau(0), z)\left(S_{i}(\tau(0)) \star_{\tau(0)} \Upsilon(0)\right)+z M(\tau(0), z)\left[z^{-1} \mathbb{S}_{i}(\tau(0))\right]_{+} \Upsilon(0) \\
& =M(\tau(0), z) \mathbb{S}_{i}(\tau(0)) \Upsilon(0)=z^{-1} \mathcal{S}_{i} \mathbf{f}(0)
\end{aligned}
$$

where we used $z \partial_{i} M(\tau, z)=M(\tau, z)\left(\phi_{i} \star_{\tau}\right)$ in the first line and Theorem 3.14 in the second line. Since the vector fields (4.2) commute each other, the corresponding vector fields $\mathbf{V}_{i}, i=1, \ldots, m$ also commute each other. In what follows, we show the existence of an integral curve for the vector field $\mathbf{V}_{i}$ with prescribed asymptotics.

Proposition 4.7. There exist unique functions

$$
\tau(y) \in H_{T}^{*}\left(X_{\Sigma}\right) \llbracket Q \rrbracket \llbracket \log y \rrbracket \quad \text { and } \quad \Upsilon(y, z) \in H_{T}^{*}\left(X_{\Sigma}\right)[z] \llbracket Q \rrbracket \llbracket \log y \rrbracket
$$

which are of the form

$$
\begin{aligned}
\tau(y) & =\sum_{i=1}^{m} u_{i} \log y_{i}+\sum_{d \in \operatorname{Eff}\left(X_{\Sigma}\right), d \neq 0} Q^{d} y^{d} \tau_{d} \\
\Upsilon(y, z) & =1+\sum_{d \in \operatorname{Eff}\left(X_{\Sigma}\right), d \neq 0} Q^{d} y^{d} \Upsilon_{d}
\end{aligned}
$$


with $y^{d}=\prod_{j=1}^{m} y_{j}^{u_{j} \cdot d}$ and give an integral curve for the vector field $\mathbf{V}_{i}$ :

$$
\frac{\partial \tau(y)}{\partial y_{i}}=S_{i}(\tau(y)) \quad \text { and } \quad \frac{\partial \Upsilon(y, z)}{\partial y_{i}}=\left[z^{-1} \mathbb{S}_{i}(\tau(y))\right]_{+} \Upsilon(y, z)
$$

for all $1 \leq i \leq m$.

Proof. Write $\tau(y)=\sum_{j=1}^{m} u_{j} \log y_{j}+\tau^{\prime}$. The divisor equation in Remark 3.12 gives

$$
\mathbb{S}_{i}(\tau(y))=y_{i}^{-1} \mathbb{S}_{i}\left(\tau^{\prime} ; Q y\right) .
$$

where $\mathbb{S}_{i}(\sigma ; Q y)$ is obtained from $\mathbb{S}_{i}(\sigma)$ by replacing $Q^{d}$ with $Q^{d} y^{d}$. Therefore we need to solve the following differential equations:

$$
y_{i} \frac{\partial \tau^{\prime}}{\partial y_{i}}=S_{i}\left(\tau^{\prime} ; Q y\right)-u_{i} \quad \text { and } \quad y_{i} \frac{\partial \Upsilon}{\partial y_{i}}=\left[z^{-1} \mathbb{S}_{i}\left(\tau^{\prime} ; Q y\right)\right]_{+} \Upsilon
$$

We expand

$$
\tau^{\prime}=\sum_{d \in \operatorname{Eff}\left(X_{\Sigma}\right), d \neq 0} \tilde{\tau}_{d}(y) Q^{d}, \quad \Upsilon=\sum_{d \in \operatorname{Eff}\left(X_{\Sigma}\right)} \widetilde{\Upsilon}_{d}(y) Q^{d}
$$

with $\widetilde{\Upsilon}_{0}(y)=1$ and solve for the coefficients $\tilde{\tau}_{d}(y), \widetilde{\Upsilon}_{d}(y)$ recursively. Note that the equation (4.3) holds true mod $Q$ by Lemma 4.5 .

First we solve for $\tau^{\prime}$. Choose a Kähler class $\omega$ such that $\omega \cdot d_{1}=\omega \cdot d_{2}$ for $d_{1}, d_{2} \in$ $\operatorname{Eff}\left(X_{\Sigma}\right)$ if and only if $d_{1}=d_{2}$. This defines a positive real grading on the Novikov ring $\mathbb{C} \llbracket Q \rrbracket$ such that $\operatorname{deg} Q^{d}=\omega \cdot d$. Take $d_{0} \in \operatorname{Eff}\left(X_{\Sigma}\right) \backslash\{0\}$. Suppose by induction that there exist $\tilde{\tau}_{d}$ for all $d$ with $\omega \cdot d<\omega \cdot d_{0}$ such that $\tilde{\tau}_{d}=\tau_{d} y^{d}$ for some $\tau_{d} \in H_{T}^{*}(X)$ and that $\tau^{\prime}=\sum_{\omega \cdot d<\omega \cdot d_{0}} \tilde{\tau}_{d} Q^{d}$ satisfies the differential equation (4.3) modulo terms of degree $\geq \omega \cdot d_{0}$. We write $\tau_{d}$ as a polynomial in $v_{1}, \ldots, v_{m}$ and $\lambda_{1}, \ldots, \lambda_{m}$. Comparing the coefficients of $Q^{d_{0}}$ of the differential equation, we obtain using Lemma 4.5 that:

$$
y_{i} \frac{\partial \tilde{\tau}_{d_{0}}}{\partial y_{i}}+u_{i} \frac{\partial \tilde{\tau}_{d_{0}}}{\partial \lambda_{i}}=\left(\begin{array}{l}
\text { an expression in } \tilde{\tau}_{d} \\
\text { with } \omega \cdot d<\omega \cdot d_{0}
\end{array}\right) .
$$

Here the right-hand side is of the form $g_{i}(v, \lambda) y^{d_{0}}$ by induction hypothesis, where $g_{i}(v, \lambda)$ is a polynomial in $v_{1}, \ldots, v_{m}$ and $\lambda_{1}, \ldots, \lambda_{m}$. Setting $\tilde{\tau}_{d_{0}}=\tau_{d_{0}} y^{d_{0}}$, we obtain

$$
\left(u_{i} \cdot d_{0}\right) \tau_{d_{0}}+\left(v_{i}+\lambda_{i}\right) \frac{\partial \tau_{d_{0}}}{\partial \lambda_{i}}=g_{i}(v, \lambda)
$$

The Kähler class can be written as a non-negative linear combination of $u_{i}$, and thus there exists $i_{0}$ such that $u_{i_{0}} \cdot d_{0}>0$. Then we can solve for the polynomial $\tau_{d_{0}}=\tau_{d_{0}}(v, \lambda)$ from the above equation with $i=i_{0}$ recursively from the highest order term in $\lambda_{i_{0}}$. Setting $\tau(y)=\sum_{i} u_{i} \log y_{i}+\sum_{\omega \cdot d \leq \omega \cdot d_{0}} \tau_{d} y^{d} Q^{d}$, we have

$$
\frac{\partial \tau(y)}{\partial y_{i}} \equiv S_{i}(\tau(y))
$$


modulo terms of degree $\geq \omega \cdot d_{0}$ for $i \neq i_{0}$ and modulo terms of degree $>\omega \cdot d_{0}$ for $i=i_{0}$. The commutativity of the flow implies that we have for $i \neq i_{0}$,

$$
\begin{aligned}
\frac{\partial}{\partial y_{i_{0}}}\left(\frac{\partial \tau}{\partial y_{i}}-S_{i}(\tau(y))\right) & =\frac{\partial^{2} \tau(y)}{\partial y_{i} \partial y_{i_{0}}}-\left(d_{\frac{\partial \tau(y)}{\partial y_{i_{0}}}} S_{i}\right)(\tau(y)) \\
& \equiv \frac{\partial S_{i_{0}}(\tau(y))}{\partial y_{i}}-\left(d_{S_{i_{0}}(\tau(y))} S_{i}\right)(\tau(y)) \\
& =\left(d_{\frac{\partial \tau(y)}{\partial y_{i}}} S_{i_{0}}\right)(\tau(y))-\left(d_{S_{i}(\tau(y))} S_{i_{0}}\right)(\tau(y)) \\
& =\left(d_{\frac{\partial \tau(y)}{\partial y_{i}}-S_{i}(\tau(y))} S_{i_{0}}\right)(\tau(y))
\end{aligned}
$$

modulo terms of degree $>\omega \cdot d_{0}$. Using the divisor equation again, we have

$$
y_{i}\left(\frac{\partial \tau(y)}{\partial y_{i}}-S_{i}(\tau(y))\right)=u_{i}+y_{i} \frac{\partial \tau^{\prime}}{\partial y_{i}}-S_{i}\left(\tau^{\prime} ; Q y\right)
$$

Modulo terms of degree $>\omega \cdot d_{0}$, this is $\alpha(Q y)^{d_{0}}$ for some $\alpha=\alpha(v, \lambda) \in H_{T}^{*}(X)$. Now the coefficient of $Q^{d_{0}}$ of equation (4.4) gives (by Lemma 4.5):

$$
\left(u_{i_{0}} \cdot d_{0}\right) \alpha+u_{i_{0}} \frac{\partial \alpha}{\partial \lambda_{i_{0}}}=0 .
$$

We want to show that $\alpha=0$ as a cohomology class. Consider the restriction $\alpha(x)$ of $\alpha$ to a $T$-fixed point $x \in X_{\Sigma}$. If $x$ lies in the divisor $\left\{z_{i_{0}}=0\right\}, v_{j}(x) \in H_{T}^{2}(\mathrm{pt})$ is a linear combination of $\lambda_{j^{\prime}}$ with $j^{\prime} \neq i_{0}$ by Lemma 4.6. Thus

$$
\left.\frac{\partial \alpha}{\partial \lambda_{i_{0}}}\right|_{x}=\frac{\partial \alpha(x)}{\partial \lambda_{i_{0}}}
$$

If $x$ is not in the divisor $\left\{z_{i_{0}}=0\right\}, u_{i_{0}}(x)=0$. Therefore, by restricting to $x$, we have

$$
\left(u_{i_{0}} \cdot d\right) \alpha(x)+u_{i_{0}}(x) \frac{\partial \alpha(x)}{\partial \lambda_{i_{0}}}=0 .
$$

This shows that $\alpha(x)=0$ recursively from the highest order term in $\lambda_{i_{0}}$. Note that the same argument shows the uniqueness of $\tau_{d_{0}}$. This completes the induction.

Next we solve for $\Upsilon$ assuming that $\tau^{\prime}$ is already solved. Let $\omega$ be a Kähler class as above and $d_{0} \in \operatorname{Eff}\left(X_{\Sigma}\right)$ be a non-zero effective class. Suppose by induction that there exist $\widetilde{\Upsilon}_{d}$ for all $d$ with $\omega \cdot d<\omega \cdot d_{0}$ such that $\widetilde{\Upsilon}_{d}=\Upsilon_{d} y^{d}$ and that $\Upsilon=\sum_{\omega \cdot d<\omega \cdot d_{0}} \widetilde{\Upsilon}_{d} Q^{d}$ satisfies the differential equation (4.3) modulo terms of degree $\geq \omega \cdot d_{0}$. We regard $\Upsilon_{d}$ as a polynomial in $v_{1}, \ldots, v_{m}$ and $\lambda_{1}, \ldots, \lambda_{m}$. Comparing the coefficients of $Q^{d_{0}}$ of the differential equation and using Lemma 4.5, we obtain

$$
y_{i} \frac{\partial \widetilde{\Upsilon}_{d_{0}}(v, \lambda)}{\partial y_{i}}-\left(v_{i}+\lambda_{i}\right) z^{-1}\left(\widetilde{\Upsilon}_{d_{0}}\left(v, \lambda-e_{i} z\right)-\widetilde{\Upsilon}_{d_{0}}(v, \lambda)\right)=\left(\begin{array}{c}
\text { an expression in } \widetilde{\Upsilon}_{d} \\
\text { with } \omega \cdot d<\omega \cdot d_{0}
\end{array}\right) .
$$

Here the right-hand side is of the form $g_{i}(v, \lambda) y^{d_{0}}$ for some polynomial $g_{i}(v, \lambda)$ in $v_{1}, \ldots, v_{m}$ and $\lambda_{1}, \ldots, \lambda_{m}$. Setting $\widetilde{\Upsilon}_{d_{0}}=\Upsilon_{d_{0}} y^{d_{0}}$, we have

$$
\left(u_{i} \cdot d_{0}\right) \Upsilon_{d_{0}}(v, \lambda)-\left(v_{i}+\lambda_{i}\right) z^{-1}\left(\Upsilon_{d_{0}}\left(v, \lambda-e_{i} z\right)-\Upsilon_{d_{0}}(v, \lambda)\right)=g_{i}(v, \lambda) .
$$


As before, we can find $i_{0}$ such that $u_{i_{0}} \cdot d_{0}>0$. We can solve for $\Upsilon_{d_{0}}(v, \lambda)$ recursively from the highest order term in $\lambda_{i_{0}}$ using this equation with $i=i_{0}$. Setting $\Upsilon=$ $\sum_{\omega \cdot d \leq \omega \cdot d_{0}} \Upsilon_{d} Q^{d}$, we have

$$
\frac{\partial \Upsilon(y)}{\partial y_{i}} \equiv\left[z^{-1} \mathbb{S}_{i}(\tau(y))\right]_{+} \Upsilon(y)
$$

modulo terms of degree $\geq \omega \cdot d_{0}$ for $i \neq i_{0}$ and modulo terms of degree $>\omega \cdot d_{0}$ for $i=i_{0}$. We have for $i \neq i_{0}$,

$$
\begin{gathered}
\frac{\partial}{\partial y_{i_{0}}}\left(\frac{\partial \Upsilon(y)}{\partial y_{i}}-\left[z^{-1} \mathbb{S}_{i}(\tau(y))\right]_{+} \Upsilon(y)\right)=\frac{\partial^{2} \Upsilon(y)}{\partial y_{i} \partial y_{i_{0}}}-\frac{\partial}{\partial y_{i_{0}}}\left[z^{-1} \mathbb{S}_{i}(\tau(y))\right]_{+} \Upsilon(y) \\
\equiv \frac{\partial}{\partial y_{i}}\left[z^{-1} \mathbb{S}_{i_{0}}(\tau(y))\right]_{+} \Upsilon(y)-\frac{\partial}{\partial y_{i_{0}}}\left[z^{-1} \mathbb{S}_{i}(\tau(y))\right]_{+} \Upsilon(y) \\
\equiv\left[z^{-1}\left(d_{S_{i}(\tau(y))} \mathbb{S}_{i_{0}}\right)(\tau(y))\right]_{+} \Upsilon(y)+\left[z^{-1} \mathbb{S}_{i_{0}}(\tau(y))\right]_{+} \frac{\partial \Upsilon(y)}{\partial y_{i}} \\
\quad-\left[z^{-1}\left(d_{S_{i_{0}}(\tau(y))} \mathbb{S}_{i}\right)(\tau(y))\right]_{+} \Upsilon(y)-\left[z^{-1} \mathbb{S}_{i}(\tau(y))\right]_{+}\left[z^{-1} \mathbb{S}_{i_{0}}(\tau(y))\right]_{+} \Upsilon(y)
\end{gathered}
$$

modulo terms of degree $>\omega \cdot d_{0}$. The commutativity of the flows $\mathbf{V}_{i}, i=1, \ldots, m$ implies for $i \neq j$,

$$
\begin{aligned}
& {\left[z^{-1}\left(d_{S_{i}(\tau)} \mathbb{S}_{j}\right)(\tau)\right]_{+} \Upsilon+\left[z^{-1} \mathbb{S}_{j}(\tau)\right]_{+}\left[z^{-1} \mathbb{S}_{i}(\tau)\right]_{+} \Upsilon} \\
& \quad=\left[z^{-1}\left(d_{S_{j}(\tau)} \mathbb{S}_{i}\right)(\tau)\right]_{+} \Upsilon+\left[z^{-1} \mathbb{S}_{i}(\tau)\right]_{+}\left[z^{-1} \mathbb{S}_{j}(\tau)\right]_{+} \Upsilon
\end{aligned}
$$

Therefore we have:

$$
\frac{\partial}{\partial y_{i_{0}}}\left(\frac{\partial \Upsilon(y)}{\partial y_{i}}-\left[z^{-1} \mathbb{S}_{i}(\tau(y))\right]_{+} \Upsilon(y)\right) \equiv\left[z^{-1} \mathbb{S}_{i_{0}}(\tau(y))\right]_{+}\left(\frac{\partial \Upsilon(y)}{\partial y_{i}}-\left[z^{-1} S_{i}(\tau(y))\right]_{+} \Upsilon(y)\right)
$$

modulo terms of degree $>\omega \cdot d_{0}$. By the divisor equation, we have

$$
y_{i}\left(\frac{\partial \Upsilon(y)}{\partial y_{i}}-\left[z^{-1} \mathbb{S}_{i}(\tau(y))\right]_{+} \Upsilon(y)\right)=y_{i} \frac{\partial \Upsilon(y)}{\partial y_{i}}-\left[z^{-1} \mathbb{S}_{i}\left(\tau^{\prime} ; Q y\right)\right]_{+} \Upsilon(y)
$$

This is of the form $\alpha(Q y)^{d_{0}}$ for some $\alpha=\alpha(v, \lambda, z) \in H_{\widehat{T}}^{*}\left(X_{\Sigma}\right)$, modulo terms of degree $>\omega \cdot d_{0}$. Hence the differential equation (4.6) implies via Lemma 4.5 that:

$$
\left(u_{i_{0}} \cdot d_{0}\right) \alpha-u_{i_{0}} z^{-1}\left(\alpha\left(v, \lambda-e_{i_{0}} z, z\right)-\alpha(v, \lambda, z)\right)=0 .
$$

We want to show that $\alpha=0$ in the cohomology group. By restricting this to a $T$-fixed point $x$ and using a similar argument as before (see (4.5)), we obtain

$$
\left(u_{i_{0}} \cdot d_{0}\right) \alpha(x)-\left(v_{i_{0}}(x)+\lambda_{i_{0}}\right) z^{-1}\left(e^{-z \partial_{\lambda_{i}}} \alpha(x)-\alpha(x)\right)=0
$$

for the restriction $\alpha(x) \in H_{\widehat{T}}^{*}(\mathrm{pt})$ of $\alpha$ to $x$. We can easily see that $\alpha(x)=0$ recursively from the highest order term in $\lambda_{i_{0}}$. Therefore $\alpha=0$. Note that the same argument also shows the uniqueness of $\Upsilon_{d_{0}}$. This completes the induction and the proof. 
We now come to the final step of the proof. Let $\tau(y), \Upsilon(y, z)$ be as in Proposition 4.7. Then, as discussed in a paragraph preceding Proposition 4.7,

$$
y \longmapsto \mathbf{f}(y):=z M(\tau(y), z) \Upsilon(y, z)
$$

defines an integral manifold for the vector fields in (4.2). We shall show that $\mathbf{f}(y)=$ $I(y, z)$. Using the divisor equation for $M(\tau, z)$, we find that $\mathbf{f}(y)$ is of the form:

$$
\mathbf{f}(y)=z e^{\sum_{i=1}^{m} u_{i} \log y_{i} / z}\left(1+\sum_{d \in \operatorname{Eff}\left(X_{\Sigma}\right) \backslash\{0\}} \mathbf{f}_{d} Q^{d} y^{d}\right)
$$

with $\mathbf{f}_{d} \in H_{\widehat{T}}(X)_{\text {loc. }}$ In view of Lemma 4.3 , the following lemma shows that $\mathbf{f}(y)=$ $I(y, z)$ and completes the proof of Theorem 4.1.

Lemma 4.8. The family of elements $y \mapsto \mathbf{f}(y)$ of the form (4.7) satisfying $\partial_{y_{i}} \mathbf{f}(y)=$ $z^{-1} \mathcal{S}_{i} \mathbf{f}(y), i=1, \ldots, m$ is unique.

Proof. Suppose that we have two families $\mathbf{f}_{1}(y), \mathbf{f}_{2}(y)$ of elements of the form (4.7) satisfying $\partial_{y_{i}} \mathbf{f}_{j}(y)=z^{-1} \mathcal{S}_{i} \mathbf{f}_{j}(y), j=1,2, i=1,2, \ldots, m$. The difference $g(y)=\mathbf{f}_{1}(y)-$ $\mathbf{f}_{2}(y)$ satisfies the same differential equation and is of the form

$$
g(y)=z e^{\sum_{i=1}^{m} u_{i} \log y_{i} / z} \sum_{d \in \operatorname{Eff}\left(X_{\Sigma}\right) \backslash\{0\}} g_{d} Q^{d} y^{d} .
$$

Choose a Kähler class $\omega$ and suppose by induction that we know $g_{d}=0$ for all $d \in$ $\operatorname{Eff}\left(X_{\Sigma}\right)$ with $\omega \cdot d<\omega \cdot d_{0}$ for some $d_{0} \in \operatorname{Eff}\left(X_{\Sigma}\right) \backslash\{0\}$. Let $x$ be a $T$-fixed point. Let $\delta$ be the set of indices $i$ such that $x$ does not lie on the toric divisor $\left\{z_{i}=0\right\}$. The Kähler class $\omega$ can be written as a positive linear combination of non-equivariant limits of $u_{i}$ with $i \in \delta$. Therefore, there exists $i_{0} \in \delta$ such that $u_{i_{0}} \cdot d_{0}>0$. The coefficient in front of $Q^{d_{0}}$ of the equation $\partial_{y_{i_{0}}} g(y)=z^{-1} \mathcal{S}_{i_{0}} g(y)$ restricted to the fixed point $x$ gives:

$$
\left(u_{i_{0}} \cdot d_{0}\right) g_{d_{0}}(x)=0
$$

since $x$ does not lie on the minimal fixed component $\left\{z_{i_{0}}=0\right\}$ with respect to $e_{i_{0}}$. Therefore $g_{d_{0}}(x)=0$. Since $x$ is arbitrary, $g_{d_{0}}=0$. This completes the induction and the proof.

4.4. Example. Consider the toric variety $X_{\Sigma}=\mathbb{P}^{m-1}$. In this case we have $m$ shift operators $\mathbb{S}_{1}, \ldots, \mathbb{S}_{m}$ corresponding to $m$ toric divisors. It is well-known that the mirror map $\tau(y)$ and the function $\Upsilon(y)$ are trivial:

$$
\tau(y)=\sum_{i=1}^{m} u_{i} \log y_{i}, \quad \Upsilon(y)=1 .
$$

Generalizing the differential equation in Lemma 4.3, we can show that

$$
\mathcal{S}_{i_{1}} \cdots \mathcal{S}_{i_{a}} I(y, z)=z \partial_{y_{i_{1}}} \cdots z \partial_{y_{i_{a}}} I(y, z)
$$


when $i_{1}, \ldots, i_{a}$ are distinct. This together with the intertwining property $\mathcal{S}_{i} \circ M(\tau, z)=$ $M(\tau, z) \circ \mathbb{S}_{i}(\tau)$ and the divisor equation $\mathbb{S}_{i}(\tau(y))=y_{i}^{-1} \mathbb{S}_{i}(0 ; Q y)$ implies:

$$
\mathbb{S}_{i_{1}}(0 ; Q y) \cdots \mathbb{S}_{i_{a}}(0 ; Q y) 1=\left.z \nabla_{u_{i_{1}}} \cdots z \nabla_{u_{i_{a}}} 1\right|_{\tau(y)}= \begin{cases}u_{i_{1}} \cdots u_{i_{a}} & \text { if } a<m \\ Q y_{1} \cdots y_{m} & \text { if } a=m\end{cases}
$$

where $i_{1}, \ldots, i_{a}$ are distinct and $\mathbb{S}_{i}(0 ; Q y)$ means $\left.\mathbb{S}_{i}(0)\right|_{Q \rightarrow Q y_{1} \cdots y_{m}}$. This determines the action of $\mathbb{S}_{i}(0)$ completely. Since the one-parameter subgroup $e_{1}+\cdots+e_{m}$ acts on $\mathbb{P}^{m-1}$ trivially, we have a relation $\mathbb{S}_{1}(\tau) \circ \cdots \circ \mathbb{S}_{m}(\tau)=Q$ by Corollary 3.16. Writing $u_{i}=v+\lambda_{i}$ for $i=1, \ldots, m$, we recover the relation:

$$
\left.\left(z \nabla_{v}+\lambda_{1}\right) \cdots\left(z \nabla_{v}+\lambda_{m}\right) 1\right|_{\tau=0}=Q
$$

in the equivariant small quantum $D$-module of $\mathbb{P}^{m-1}$.

4.5. Remarks. We first remark a relation to the results in GI12. Let $X_{\Sigma}$ be a compact toric manifold such that $c_{1}\left(X_{\Sigma}\right)$ is nef. In this case, the mirror map $\tau(y)$ takes values in $H_{T}^{2}(X)$. We write

$$
\tau(y)=\sum_{i=1}^{m}\left(\log y_{i}-g^{i}(y)\right) u_{i}
$$

for some $\mathbb{C}$-valued functions $g^{i}(y)$. Using the divisor equation from Remark 3.12, the differential equation in Proposition 4.7 implies:

$$
y_{i} \frac{\partial \tau(y)}{\partial y_{i}}=e^{g^{i}(y)} S_{i}\left(0 ; Q e^{\tau(y)}\right)
$$

where we set $S_{i}\left(0 ; Q e^{\tau(y)}\right)=\left.S_{i}(0)\right|_{Q \rightarrow Q e^{\tau(y)}}$. The left-hand side is called the Batyrev element in GI12 and this recovers the relationship between the Seidel and the Batyrev elements in GI12, Theorem 1.1].

We should also recover a mirror theorem for the extended $I$-function CCIT13 by considering the shift operators corresponding to general semi-negative cocharacters $k \in$ $\left(\mathbb{Z}_{\geq 0}\right)^{m} \subset \operatorname{Hom}\left(\mathbb{C}^{\times}, T\right)$. It would be also interesting to see if our method can be generalized to toric orbifolds [CCIT13, CCFK14], toric fibrations [Bro09], or other $T$ varieties.

\section{REFERENCES}

[AB84] M. F. Atiyah and R. Bott. The moment map and equivariant cohomology. Topology, 23(1):1-28, 1984.

[Aud04] Michèle Audin. Torus actions on symplectic manifolds, volume 93 of Progress in Mathematics. Birkhäuser Verlag, Basel, revised edition, 2004.

[BB73] A. Białynicki-Birula. Some theorems on actions of algebraic groups. Ann. of Math. (2), 98:480-497, 1973.

[BMO11] Alexander Braverman, Davesh Maulik, and Andrei Okounkov. Quantum cohomology of the Springer resolution. Adv. Math., 227(1):421-458, 2011.

[Bro09] Jeffrey Steven Brown. Gromov-Witten invariants of toric fibrations. 2009. arXiv:0901.1290 [math.AG], to appear in IMRN.

[CCFK14] Daewoong Cheong, Ionut Ciocan-Fontanine, and Bumsig Kim. Orbifold quasimap theory. arXiv:1405.7160 [math.AG], 2014. 
[CCIT13] Tom Coates, Alessio Corti, Hiroshi Iritani, and Hsian-Hua Tseng. A mirror theorem for toric stacks. arXiv:1310.4163 [math.AG], 2013.

[CG07] Tom Coates and Alexander Givental. Quantum Riemann-Roch, Lefschetz and Serre. Ann. of Math. (2), 165(1):15-53, 2007.

[CIJ14] Tom Coates, Hiroshi Iritani, and Yunfeng Jiang. The crepant transformation conjecture for toric complete intersections. 2014. arXiv:1410.0024 [math.AG].

[CK99] David A. Cox and Sheldon Katz. Mirror symmetry and algebraic geometry, volume 68 of Mathematical Surveys and Monographs. American Mathematical Society, Providence, RI, 1999.

[CLS11] David A. Cox, John B. Little, and Henry K. Schenck. Toric varieties, volume 124 of Graduate Studies in Mathematics. American Mathematical Society, Providence, RI, 2011.

[Dol03] Igor Dolgachev. Lectures on invariant theory, volume 296 of London Mathematical Society Lecture Note Series. Cambridge University Press, Cambridge, 2003.

[GI12] Eduardo González and Hiroshi Iritani. Seidel elements and mirror transformations. Selecta Math. (N.S.), 18(3):557-590, 2012.

[Giv98a] Alexander Givental. Elliptic Gromov-Witten invariants and the generalized mirror conjecture. In Integrable systems and algebraic geometry (Kobe/Kyoto, 1997), pages 107-155. World Sci. Publ., River Edge, NJ, 1998.

[Giv98b] Alexander Givental. A mirror theorem for toric complete intersections. In Topological field theory, primitive forms and related topics (Kyoto, 1996), volume 160 of Progr. Math., pages 141-175. Birkhäuser Boston, Boston, MA, 1998.

[Giv04] Alexander Givental. Symplectic geometry of Frobenius structures. In Frobenius manifolds, Aspects Math., E36, pages 91-112. Friedr. Vieweg, Wiesbaden, 2004.

[GP99] Tom Graber and Rahul Pandharipande. Localization of virtual classes. Invent. Math., 135(2):487-518, 1999.

[HRV13] Tamas Hausel and Fernando Rodriguez-Villegas. Cohomology of large semiprojective hyperKähler varieties. arXiv:1309.4914 [math.AG], 2013.

[Iri08] Hiroshi Iritani. Quantum $D$-modules and generalized mirror transformations. Topology, 47(4):225-276, 2008.

[Iri09] Hiroshi Iritani. An integral structure in quantum cohomology and mirror symmetry for toric orbifolds. Adv. Math., 222(3):1016-1079, 2009.

[Kir84] Frances Clare Kirwan. Cohomology of quotients in symplectic and algebraic geometry, volume 31 of Mathematical Notes. Princeton University Press, Princeton, NJ, 1984.

[KKP08] Ludmil Katzarkov, Maxim Kontsevich, and Tony Pantev. Hodge theoretic aspects of mirror symmetry. In From Hodge theory to integrability and TQFT tt*-geometry, volume 78 of Proc. Sympos. Pure Math., pages 87-174. Amer. Math. Soc., Providence, RI, 2008.

[LLY99] Bong H. Lian, Kefeng Liu, and Shing-Tung Yau. Mirror principle. III. Asian J. Math., 3(4):771-800, 1999.

[MO12] Davesh Maulik and Andrei Okounkov. Quantum groups and quantum cohomology. arXiv:1211.1287 [math.AG], 2012.

[MT06] Dusa McDuff and Susan Tolman. Topological properties of Hamiltonian circle actions. IMRP Int. Math. Res. Pap., pages 72826, 1-77, 2006.

[Nak99] Hiraku Nakajima. Lectures on Hilbert schemes of points on surfaces, volume 18 of University Lecture Series. American Mathematical Society, Providence, RI, 1999.

[Oda88] Tadao Oda. Convex bodies and algebraic geometry, volume 15 of Ergebnisse der Mathematik und ihrer Grenzgebiete (3) [Results in Mathematics and Related Areas (3)]. Springer-Verlag, Berlin, 1988. An introduction to the theory of toric varieties, Translated from the Japanese.

[OP10] Andrei Okounkov and Rahul Pandharipande. The quantum differential equation of the Hilbert scheme of points in the plane. Transform. Groups, 15(4):965-982, 2010.

[Pan98] Rahul Pandharipande. Rational curves on hypersurfaces (after A. Givental). Astérisque, (252):Exp. No. 848, 5, 307-340, 1998. Séminaire Bourbaki. Vol. 1997/98. 
[Sei97] Paul Seidel. $\pi_{1}$ of symplectic automorphism groups and invertibles in quantum homology rings. Geom. Funct. Anal., 7(6):1046-1095, 1997. arXiv:dg-ga/9511011.

E-mail address: iritani@math.kyoto-u.ac.jp

Department of Mathematics, Graduate School of Science, Kyoto University, KitashirakaWA-OiWAKe-CHO, SAKYO-KU, KYOto, 606-8502, JAPAN 